\title{
BIAS CORRECTION OF SEMIPARAMETRIC LONG MEMORY PARAMETER ESTIMATORS VIA THE PREFILTERED SIEVE BOOTSTRAP
}

\author{
D. S. Poskitt, Gael M. Martin and Simone D. Grose \\ Monash University
}

\begin{abstract}
This paper investigates bootstrap-based bias correction of semiparametric estimators of the long memory parameter, $d$, in fractionally integrated processes. The resampling method involves the application of the sieve bootstrap to data prefiltered by a preliminary semiparametric estimate of the long memory parameter. Theoretical justification for using the bootstrap technique to bias adjust log periodogram and semiparametric local Whittle estimators of the memory parameter is provided in the case where the true value of $d$ lies in the range $0 \leq d<0.5$. That the bootstrap method provides confidence intervals with the correct asymptotic coverage is also proven, with the intervals shown to adjust explicitly for bias, as estimated via the bootstrap. Simulation evidence comparing the performance of the bootstrap bias correction with analytical bias-correction techniques is presented. The bootstrap method is shown to produce notable bias reductions, in particular when applied to an estimator for which some degree of bias reduction has already been accomplished by analytical means.
\end{abstract}

\section{INTRODUCTION}

The so-called long memory, or strongly dependent, processes have come to play an important role in time series analysis. Long-range dependence, observed in a very wide range of empirical applications, is characterized by an autocovariance structure that decays too slowly to be absolutely summable. Specifically, rather than the autocovariance function declining at the exponential rate characteristic of a stable and invertible ARMA process, it declines at a hyperbolic rate dependent on a "long memory" parameter. A detailed description of the properties of such processes can be found in Beran (1994). Perhaps the most popular model of a long memory process is the fractionally integrated $(I(d))$ process introduced by Granger and Joyeux (1980) and Hosking (1981). This class of processes can be characterized by the specification,

This research has been supported by Australian Research Council (ARC) Discovery Grant DP120102344. The authors would like to thank the Editor, a co-editor and two referees for very detailed and constructive comments on earlier drafts of the paper. Address correspondence to Gael Martin, Department of Econometrics and Business Statistics, Monash University, Victoria 3800, Australia; e-mail: gael.martin@ monash.edu. 
$y(t)=\sum_{j=0}^{\infty} k(j) \varepsilon(t-j)=\frac{\kappa(z)}{(1-z)^{d}} \varepsilon(t)$,

where $\varepsilon(t)$ is zero-mean white noise, $z$ is here interpreted as the lag operator $\left(z^{j} y(t)=y(t-j)\right)$, and $\kappa(z)=\sum_{j \geq 0} \kappa(j) z^{j}, \kappa(0)=1$. For any $d>-1$ the operator $(1-z)^{d}$ is defined via a binomial expansion and if the "short memory" component $\kappa(z)$ is the transfer function of a stable, invertible ARMA process and $|d|<0.5$, then the coefficients of $k(z)$ are square-summable $\left(\sum_{j \geq 0}|k(j)|^{2}<\infty\right)$. In this case $y(t)$ is well-defined as the limit in mean square of a covariancestationary process and the model is essentially a generalization of the classic BoxJenkins ARIMA model (Box and Jenkins, 1970),

$(1-z)^{d} \Phi(z) y(t)=\Theta(z) \varepsilon(t)$

in which we now allow noninteger values of the integrating parameter $d$ and $\kappa(z)=\Theta(z) / \Phi(z)$.

The long-run behaviour of the process in (2) naturally depends on the fractional integration parameter $d$. In particular, for any $d>0$ the impulse response coefficients of the Wold representation in (1) are not absolutely summable and, for $0<d<0.5$, the autocovariances decline at the rate $\gamma(\tau) \sim C \tau^{2 d-1}$. Such processes have been found to exhibit dynamic behaviour very similar to that observed in many empirical time series. See Robinson (2003) for a collection of the seminal articles in the area and Doukhan, Oppenheim, and Taqqu (2003) for a thorough review of theory and applications.

Statistical procedures for analyzing long memory processes have ranged from the likelihood-based methods of Fox and Taqqu (1986), Dahlhaus (1989), Sowell (1992) and Beran (1995), to the semiparametric techniques advanced by Geweke and Porter-Hudak (1983) and Robinson (1995a,1995b), among others. The asymptotic theory for maximum likelihood estimation of the parameters of such processes is well established, at least under the assumption of Gaussian errors. In particular, we have consistency, asymptotic efficiency, and asymptotic normality for the MLE of the fractional differencing parameter, so providing a basis for large sample inference in the usual manner. Such asymptotic results are, however, conditional on correct model specification, with the MLE of $d$ typically inconsistent if either or both the autoregressive and moving average operators in (2) (or, equivalently, the operator $\kappa(z)$ in (1)) are incorrectly specified. The semiparametric methods aim to produce consistent estimators of $d$ while placing only very mild restrictions on the behaviour of $\kappa\left(e^{\imath \lambda}\right)$ for frequency values $\lambda$ near zero. The semiparametric estimators are therefore robust to different forms of short-run dynamics and offer broader applicability than a fully parametric method. They are also asymptotically pivotal and have particularly simple asymptotic normal distributions.

Whilst such features place the semiparametric methods at the forefront for use in conducting inference on $d$, the price paid for their application is a reduction in asymptotic efficiency (relative to exact ML) and a slower rate of convergence to 
the true parameter (Giraitis, Robinson, and Samarov 1997). Also, despite asymptotic robustness to the short-run dynamics, semiparametric estimators have been shown to exhibit large finite sample bias in the presence (in particular) of a substantial autoregressive component - see Agiakloglou, Newbold, and Wohar (1993) and Lieberman (2001) for examples. In response to these findings, analytical approaches to reducing the first-order bias of semiparametric estimators have been proposed. Moulines and Soulier (1999), for example, reduce bias by fitting a finite number of Fourier coefficients to the logarithm of the short memory spectrum and constructing a broad-band log periodogram regression (LPR) estimator of $d$ that uses all of the frequencies in the range $(0, \pi]$, not just those in a neighborhood of zero. Andrews and Guggenberger (2003) consider a biasadjusted estimator of $d$ obtained by including even powers of frequency as additional regressors in the pseudo regression that defines the LPR estimator, and Andrews and Sun (2004) adapt this approach to the semiparametric local Whittle (SPLW) estimator examined in Robinson (1995a).

As a point of contrast with existing work, the focus of this paper is on the use of the bootstrap to bias correct semiparametric estimators of the long memory parameter. As is consistent with the semiparametric approach to estimation of $d$ itself, a semiparametric approach to the bootstrap scheme is also adopted, based on the "sieve" technique. This works by "prewhitening" the data using an autoregressive approximation, with the dynamics of the process captured in a fitted autoregression (see Politis, 2003). Provided the order, $h$, of the autoregression increases at a suitable rate with $T$, the convergence rates for the sieve bootstrap are much closer (in fact arbitrarily close) to those for simple random samples. Choi and Hall (2000) demonstrate the superior convergence performance of the sieve bootstrap over the block bootstrap for linear short memory processes, while Poskitt, Grose, and Martin (2015) build on the results of Poskitt (2008) to show that under regularity conditions that allow for $I(d)$ processes the sieve bootstrap achieves an error rate of $O_{p}\left(T^{-(1-\max \{0, d\})+\beta}\right), \beta>0$, for the quantiles of the sampling distribution of a general class of statistics that includes the sample mean and second-order moments.

The current paper uses a modified sieve bootstrap, wherein a consistent semiparametric estimator of the long memory parameter is used to prefilter the raw data, prior to the use of a long autoregressive approximation as the sieve from which bootstrap samples are produced. As the focus of the paper is on the use of the bootstrap to bias correct, theoretical results are presented that pertain directly to the accuracy with which the prefiltered sieve method estimates the true bias in the relevant estimators of $d$. Specifically, we derive error rates for bootstrapbased estimation of the bias of $\sqrt{N}-\mathrm{CAN}$ (consistent and asymptotically normal); $N \sim K T^{v}, K \in(0, \infty), 0<v<1$; estimators that satisfy a requisite Edgeworth expansion, subject to the prefiltering value itself converging almost surely to the true value of $d$ at a sufficient rate. The theoretical validity of bootstrap highest probability density (HPD) confidence intervals constructed from the prefiltered bootstrap replications is also established. To demonstrate the bootstrap technique we use it 
to bias correct the LPR and SPLW estimators, plus the analytically-bias-adjusted variants of Andrews and Guggenberger (2003) and Andrews and Sun (2004).

Our exposition centers around the short and long memory stationary case, with the true value of $d$ assumed to lie in the range $0 \leq d<0.5$. Whilst this may be deemed to be a limitation of sorts, our key theoretical results are stated in a form that suggests that they will have more general applicability, subject only to the proviso that the assumption of stationarity can be relaxed to accommodate more general processes. For example, nonstationary long memory structures could be catered for by considering data generating mechanisms driven by fractional noise of the form

$n(t)= \begin{cases}\sum_{s=0}^{t-1} \alpha_{s}^{(d)} \varepsilon(t-s), & d \in[0,0.5) ; \\ \sum_{s=0}^{t-1} \sum_{\tau=0}^{t-s-1} \alpha_{\tau}^{(1-d)} \varepsilon(t-s-\tau), & d \in[0.5,1.5),\end{cases}$

where $\alpha_{s}^{(d)}, s=0,1,2, \ldots$, denote the coefficients of the fractional difference operator when expressed in terms of its binomial expansion, as in equation (3) below. The prefiltered sieve bootstrap could then be applied as described in Section 2 using an appropriate estimator, such as the quasi (Gaussian) maximum likelihood estimator of Tanaka (1999) or the exact local Whittle estimator of Shimotsu and Phillips (2005). The difficulty here lies not in the practical implementation of the prefiltered sieve bootstrap for such estimators, but in showing that a largedeviations condition necessary to establish the theoretical validity of the method holds - we will return to a brief discussion of this issue below.

In addition to the theoretical results, an extensive simulation exercise is undertaken, in which the bias and mean squared error (MSE) of the bootstrapbias-adjusted estimators is documented, in comparison with the corresponding statistics both for the original unadjusted estimators, and the estimators that are adjusted by analytical methods alone. As a benchmark for the effectiveness of the bias correction we also present bias and MSE results for the correctly specified (and hence asymptotically optimal) MLE. The bootstrap bias adjustment is implemented both as a one-step exercise, and as an iterative procedure, with a stochastic stopping rule invoked to produce the final estimator. The empirical coverage (and average length) of the HPD confidence intervals is also recorded for all estimators. In accordance with the theoretical results, we investigate the (relative) performance of the bootstrap bias adjustment using values of $d$ in the range $0 \leq d<0.5$ to generate the data in the simulation experiments. The LPR and SPLW estimators themselves however - both in unadjusted and bias-adjusted form - are essentially left unconstrained, as there is nothing in the structure of the prefiltered sieve bootstrap algorithm per se that requires that the estimator that is to be bias corrected, or the prefilter, be restricted to lie in the 0 to 0.5 range. $^{1}$

The paper proceeds as follows. Section 2 briefly summarizes the statistical properties of long memory processes, and outlines the prefiltered sieve bootstrap in this context. The bootstrap-based bias-adjustment algorithm is also described in this section. In Section 3 we present the key theoretical results, namely the almost 
sure convergence of the bootstrap estimator of the true bias, and the correctness of the (asymptotic) coverage probability of the bootstrap confidence intervals. The associated proofs are assembled in Appendix A. Section 4 outlines the iterated version of the bootstrap bias-adjustment technique, with details of the stochastic stopping rules invoked therein given in Appendix B. Details and discussion of the simulation study follow in Section 5. Selected simulation results are tabulated in Appendix $\mathrm{C}$, with additional tables included in an extended version of the paper, available at: http://arxiv.org/abs/1603.01897. Section 6 concludes the paper with a summary of our contribution and a discussion of the extension of the prefiltered sieve bootstrap methodology to more general processes.

\section{LONG MEMORY PROCESSES, AUTOREGRESSIVE APPROXIMATION, AND THE PREFILTERED SIEVE BOOTSTRAP}

Let $y(t)$ for $t \in \mathcal{Z}$ denote a linearly regular, covariance-stationary process, with representation as in (1), where:

Assumption 1. The transfer function in the representation (1) is given by $k(z)=\kappa(z) /(1-z)^{d}$ where $d \in[0,0.5)$ and $\kappa(z) \neq 0,|z| \leq 1$. The impulse response coefficients of $\kappa(z)$ satisfy $k(0)=1$ and $\sum_{j \geq 0} j|\kappa(j)|<\infty$.

Assumption 2. The innovations process $\varepsilon(t)$ is an i.i.d. zero mean Gaussian white noise process with variance $\sigma^{2}$.

Assumption 1 serves to characterize the spectral features of quite a wide class of short and long memory processes, including long-range dependent members of the ARFIMA family of models that are the focus of this paper. This assumption implies that the innovations in (1) are fundamental; meaning that $\varepsilon(t)$ lies in the space spanned by current and past values of $y(t)$, and $\varepsilon(t)$ and $y(s)$ are uncorrelated for all $s<t$. For a discussion of the role of fundamentalness in the context of the autoregressive sieve bootstrap see Kreiss, Paparoditis, and Politis (2011). Note that the regularity conditions employed in Kreiss et al. (2011) exclude fractional time series, but using the extension of Baxter's inequality to long-range dependent processes due to Inoue and Kasahara (2006) it is possible to generalize the results of Kreiss et al. (2011) to time series generated from a fractional transformation of a linear process. In particular, since the statistics that we investigate are asymptotically pivotal the results in Kreiss et al. (2011, Section 3) can be extended to the statistics and class of processes under consideration here.

Assumptions 1 and 2 imply that $y(t)$ is a Gaussian linear process. A basic property of a linear process that underlies the sieve bootstrap methodology and the associated results is that $y(t)$ is linearly regular and the linear predictor $\bar{y}(t)=\sum_{j=1}^{\infty} \pi(j) y(t-j)$, where $\sum_{j=0}^{\infty} \pi(j) z^{j}=(1-z)^{d} \kappa(z)^{-1}$, is the minimum mean squared error predictor of $y(t)$ based upon its entire past. The need to invoke Gaussianity is unfortunate but is unavoidable here as we wish to employ certain results from the existing literature where the assumption that $y(t)$ is a 
Gaussian process is adopted. The use of these results is made explicit in Section 3. It is likely that our results can be extended to more general linear processes, although the regularity conditions and manipulations needed for such extensions are liable to be relatively involved. Fay, Moulines, and Soulier (2004), for example, provide a discussion of Edgeworth expansions in the context of linear statistics applied to long-range dependent linear processes where the innovations process is i.i.d. zero mean white noise with variance $\sigma^{2}$, but Gaussianity is replaced by a strengthening of the Cramér condition on the characteristic function of the innovations. Extensions of the results in Fay et al. (2004) to the LPR estimator are presented in Fay (2010). ${ }^{2}$

The minimum mean squared error predictor of $y(t)$ based only on a finite number $h$ of past observations $(\operatorname{MMSEP}(h))$ is $\bar{y}_{h}(t)=\sum_{j=1}^{h} \pi_{h}(j) y(t-j) \equiv$ $-\sum_{j=1}^{h} \phi_{h}(j) y(t-j)$, where the minor reparameterization from $\pi_{h}$ to $\phi_{h}$ allows us, on also defining $\phi_{h}(0)=1$, to conveniently write the corresponding prediction error in the form of an autoregression of order $h(A R(h))$, namely $\varepsilon_{h}(t)=\sum_{j=0}^{h} \phi_{h}(j) y(t-j)$. The finite-order autoregressive coefficients $\phi_{h}(1), \ldots, \phi_{h}(h)$ can be derived from the Yule-Walker equations $\sum_{j=0}^{h} \phi_{h}(j) \gamma(j-k)=\delta_{0}(k) \sigma_{h}^{2}, \quad k=0,1, \ldots, h$; in which $\gamma(\tau)=\gamma(-\tau)=$ $E[y(t) y(t-\tau)], \tau=0,1, \ldots$, is the autocovariance function of the process $y(t)$, $\delta_{0}(k)$ is Kronecker's delta (i.e., $\delta_{0}(k)=0 \forall k \neq 0 ; \delta_{0}(0)=1$ ), and the minimum mean squared error is $\sigma_{h}^{2}=E\left[\varepsilon_{h}(t)^{2}\right]$, the prediction error variance associated with $\bar{y}_{h}(t)$.

The use of autoregressive models of finite order $h$ to approximate an unknown (but suitably regular) process therefore requires that the optimal predictor $\bar{y}_{h}(t)$ determined from the $A R(h)$ model be a good approximation to the "infiniteorder" predictor $\bar{y}(t)$ for sufficiently large $h$. The asymptotic validity and properties of $A R(h)$ models when $h \rightarrow \infty$ with the sample size $T$, under regularity conditions that admit long-range dependent processes, were established in Poskitt (2007), and we refer the reader to that paper for more details. That the sieve bootstrap, which uses an $A R(h)$ approximation to capture the dynamics of $y(t)$ (with $h$ selected optimally) is, accordingly, a plausible semiparametric bootstrap for a long memory process, was subsequently established in Poskitt (2008). We focus in this paper on a modified version of this form of bootstrap. ${ }^{3}$

\subsection{The prefiltered sieve bootstrap}

Let $\alpha_{j}^{(d)} ; d \in[0,0.5) ; j=0,1,2, \ldots$; denote the coefficients of the binomial expansion of the fractional difference operator $(1-z)^{d}=\sum_{j=0}^{\infty} \alpha_{j}^{(d)} z^{j}$,

$\alpha_{j}^{(d)}=\frac{\Gamma(j-d)}{\Gamma(-d) \Gamma(j+1)} ; j=0,1,2, \ldots$

The prefiltered sieve bootstrap (PFSB) realizations of $y(t)$ are generated using the following algorithm: 
1. For a given preliminary value $d^{f}$ of $d$ calculate the coefficients of the filter $(1-z)^{d^{f}}$, and from the observed data generate the filtered series

$$
w^{f}(t)=\sum_{j=0}^{t-1} \alpha_{j}^{\left(d^{f}\right)} y(t-j), \quad t=1, \ldots, T .
$$

2. Fit an autoregression to $w^{f}(t)$ and generate a sieve bootstrap sample $w^{*} f(t)$, $t=1, \ldots, T$, of the filtered data as follows:

(a) Given the filtered series $w^{f}(t), t=1, \ldots, T$, calculate the parameter estimates, $\bar{\phi}_{h}(1), \ldots, \bar{\phi}_{h}(h)$ and $\hat{\sigma}_{h}^{2}$, of the $A R(h)$ approximation, and evaluate the residuals $\bar{\varepsilon}_{h}(t)=\sum_{j=0}^{h} \bar{\phi}_{h}(j) w^{f}(t-j), t=1, \ldots, T$, using $w^{f}(1-j)=w^{f}(T-j+1), j=1, \ldots, h$, as initial values.

(b) Construct the standardized residuals $\tilde{\varepsilon}_{h}(t)=\left(\bar{\varepsilon}_{h}(t)-\bar{\varepsilon}_{h}\right) / \bar{\sigma}_{h}$, $t=1, \ldots, T$, where $\bar{\varepsilon}_{h}=T^{-1} \sum_{t=1}^{T} \bar{\varepsilon}_{h}(t)$ and $\bar{\sigma}_{h}^{2}=T^{-1} \sum_{t=1}^{T}\left(\bar{\varepsilon}_{h}(t)\right.$ $\left.-\bar{\varepsilon}_{h}\right)^{2}$.

(c) Set $\varepsilon_{h}^{*}(t)=\bar{\sigma}_{h} e(t), t=1, \ldots, T$, where $e(t), t=1, \ldots, T$, denotes a simple random sample of $T$ i.i.d. values drawn from the standard normal distribution.

(d) Construct the sieve bootstrap realization $w^{*_{f}}(1), \ldots, w^{*}(T)$ using the autoregressive process $\sum_{j=0}^{h} \bar{\phi}_{h}(j) w^{* f}(t-j)=\varepsilon_{h}^{*}(t)$, $t=1, \ldots, T$, initiated at $w^{*} f(1-j)=w^{f}(\tau-j+1), j=1, \ldots, h$, where $\tau$ has the discrete uniform distribution on the integers $h, \ldots, T$.

3. Using the coefficients of the (inverse) filter $(1-z)^{-d^{f}}$, construct the corresponding prefiltered sieve bootstrap draw of the process $y^{* f}(t)=$ $\sum_{j=0}^{t-1} \alpha_{j}^{\left(-d^{f}\right)} w^{* f}(t-j), t=1, \ldots, T$.

The basic, or 'raw', sieve bootstrap is equivalent to setting $d^{f}=0$ in the PFSB; in which case Steps 1 and 3 are redundant and Step 2 is applied to the raw data $y(t)$.

The properties of the raw sieve bootstrap for fractional processes are given in Poskitt (2008). Crucially, if $\bar{\phi}_{h}(z)=\sum_{j=0}^{h} \bar{\phi}_{h}(j) z^{j}$ denotes the estimator of $\phi_{h}(z)=\sum_{j=0}^{h} \phi_{h}(j) z^{j}$ when the sieve bootstrap is applied to the raw data, and $y(t)$ is a linearly regular, covariance-stationary process that satisfies Assumptions 1 and 2 , then for all $h \leq H_{T}=a(\log T)^{c}, a>0, c<\infty, \sum_{j=1}^{h}\left|\bar{\phi}_{h}(j)-\phi_{h}(j)\right|^{2}=$ $O\left(h(\log T / T)^{1-2 \max \{0, d\}}\right)$ a.s. (See also Theorem 5 and Corollary 1 of Poskitt, 2007, and the associated discussion.) Given that the order of magnitude of $\left|\bar{\phi}_{h}(z)-\phi_{h}(z)\right|$ is a function of the fractional integration parameter, it is apparent that convergence of the algorithm must depend on the values of $d^{f}$ and $d .4$

Now, when $d^{f} \neq 0,(1-z)^{d^{f}} y(t)=(1-z)^{d^{f}-d} \kappa(z) \varepsilon(t)$ has fractional index $d-d^{f}$, where by assumption $\left|d^{f}-d\right|=o(1)$ a.s.; i.e., the prefiltering value presumed to be estimated from the data, and denoted hereafter by $d^{f}=d_{T}^{f}$ 
accordingly - is assumed to be a strongly consistent estimator of $d$. Hence, for any $\delta>0$ the event $\left(d_{T}^{f}-d\right) \in N_{\delta}=\{x:|x|<\delta\}$ will occur with probability one as $T \rightarrow \infty$. The error in the AR approximation to $w^{f}(t)$ will accordingly be of order $O\left(h(\log T / T)^{1-2 \delta}\right)$ or smaller. In Section 3 it is shown that $d_{T}^{f}$ needs to satisfy the large deviations property $\left|d_{T}^{f}-d\right| \log T \rightarrow 0$ a.s. as $T \rightarrow \infty$ in order for this level of accuracy to be transferred to the prefiltered sieve bootstrap realizations $y^{* f}(t)$ of $y(t)$, via the sieve bootstrap draws $w^{* f}(t)$ of $w^{f}(t)$. Theoretical results pertaining to the accuracy of the PFSB algorithm as a mechanism for bias reduction of semiparametric long memory parameter estimators are then provided.

\subsection{Bias correction via the prefiltered sieve bootstrap}

With the conditions on $d_{T}^{f}$ verified in any particular case, we employ the prefiltered sieve bootstrap for the purpose of bias correcting the LPR and SPLW estimators of the memory parameter, and their analytically adjusted variants.

To bias correct any chosen estimator, $\widehat{d}_{T}$, of $d$ we proceed as follows:

1. Calculate $\widehat{d}_{T}$ from the data $y(t), t=1, \ldots, T$.

2. Using an appropriate data-based prefiltering value $d^{f}=d_{T}^{f}$, produce $B$ bootstrap realizations $y_{b}^{*}(t), t=1, \ldots, T ; b=1,2, \ldots, B$; of the process $y(t)$. From these construct $B$ bootstrap values of the estimator, $\widehat{d}_{T, b}^{* f}$, $b=1,2, \ldots, B$, by evaluating the estimator $\widehat{d}_{T}$ for each of the $B$ independent bootstrap draws.

3. Estimate the bias of $\widehat{d}_{T}$ by

$\widehat{b}_{T, B}^{* f}=\bar{d}_{T, B}^{* f}-d_{T}^{f}$,

where

$\bar{d}_{T, B}^{* f}=B^{-1} \sum_{b=1}^{B} \widehat{d}_{T, b}^{* f}$

and produce the bias-adjusted estimator

$\widetilde{d}_{T}=\widehat{d}_{T}-\widehat{b}_{T, B}^{* f}$.

While there is no fundamental requirement that the prefiltering value correspond to the estimator being bias corrected, this correspondence is a natural one to adopt. As such, $d_{T}^{f}$ is initially taken to represent either the LPR or SPLW estimator, with or without analytical bias adjustment, according to whichever of these estimators is the subject of bias correction. With the introduction of an iterative version of this bias-correction procedure in Section 4, the set of prefilters is expanded to include bootstrap-based bias-corrected versions of the base estimators. The validity of all such versions of the prefilter is established in the following section. 


\section{KEY THEORETICAL RESULTS}

\subsection{Convergence of the bootstrap estimator of bias}

Motivated by a consideration of the properties of the LPR and SPLW estimators that are the focus herein, suppose that $\widehat{d}_{T}$ (the estimator to be bias corrected) is an asymptotically pivotal $\sqrt{N}$-CAN estimator of $d$ where $N \sim K T^{v}, K \in(0, \infty)$, and, following Hurvich, Deo, and Brodsky (1998) and Giraitis and Robinson (2003), wherein Gaussianity is assumed as it is in the current paper, we now restrict $v$ to lie in the range $(2 / 3,4 / 5)$. Let $b_{T}$ denote the finite sample bias of $\widehat{d}_{T}$, that is,

$b_{T}=E\left[\widehat{d}_{T}\right]-d$.

Here $E$ denotes expectation taken with respect to the original probability space $(\Omega, \mathfrak{F}, P)$. Now let $\widehat{d}_{T}^{* f}$ denote the value of $\widehat{d}_{T}$ calculated from a bootstrap realization of the process, $y^{*} f(t), t=1, \ldots, T$, constructed using the PFSB algorithm where the prefiltering value $d_{T}^{f}$ by construction satisfies the conditions stated above for $\widehat{d}_{T}$, given that we equate $d_{T}^{f}$ to $\widehat{d}_{T}$ in any particular instance. Since the process $\varepsilon_{h}^{*}(t)$ is Gaussian, it follows that $y^{*} f(t)$ will be a fractionally integrated $A R(h)$ Gaussian process with parameters $d_{T}^{f}$ and $\bar{\phi}_{h}(1), \ldots, \bar{\phi}_{h}(h)$, and $\widehat{d}_{T}^{* f}$ is a $\sqrt{N}$-CAN estimator of $d_{T}^{f}$. Proceeding as previously, replacing $\widehat{d}_{T}$ by $\widehat{d}_{T}^{* f}, d$ by $d_{T}^{f}$ and $E\left[\widehat{d}_{T}\right]$ by $E^{*}\left[\widehat{d}_{T}^{* f}\right]$, we have

$b_{T}^{*}=E^{*}\left[\widehat{d}_{T}^{* f}\right]-d_{T}^{f}$,

where $E^{*}$ denotes the expectation associated with $\left(\Omega^{*}, \mathfrak{F}^{*}, P^{*}\right)$, the probability space induced by the bootstrap process. Given that $\bar{d}_{T, B}^{* f}$ in (6) can be made arbitrarily close to $E^{*}\left[\widehat{d}_{T}^{* f}\right]$ by taking $B$ sufficiently large, (9) represents the estimator of the true finite sample bias induced by the prefiltered sieve bootstrap.

The accuracy with which $b_{T}^{*}$ estimates $b_{T}$ obviously underpins the validity of the bootstrap bias correction method. To evaluate the magnitude of $\left|b_{T}-b_{T}^{*}\right|$ note that $\left|\kappa\left(e^{l \lambda}\right)\right|^{2}$ (for $\kappa(\cdot)$ as defined in (1)) is a bounded, even function of $\lambda$, and we have the power series (McLaurin) expansion $\left|\kappa\left(e^{\imath \lambda}\right)\right|^{2}=c_{0}+\sum_{j \geq 1} c_{j}|\lambda|^{2 j}=$ $c_{0}+c_{1}|\lambda|^{2}+o\left(|\lambda|^{3}\right)$ as $|\lambda| \rightarrow 0$. Then, as is shown in Hurvich et al. (1998) and Giraitis and Robinson (2003), see also Andrews and Guggenberger (2003) and Andrews and Sun (2004),

$b_{T}=-\beta \frac{2 c_{1}}{9 c_{0}}\left(\frac{N}{T}\right)^{2}+o\left(\frac{N^{2}}{T^{2}}\right)$

for the LPR and SPLW estimators, where $\beta>0$. Similarly, set $\bar{\kappa}_{h}(z)=$ $\sum_{j=0}^{\infty} \bar{\kappa}_{h}(j) z^{j}$, where the $\bar{\kappa}_{h}(j)$ and $\bar{\phi}_{h}(j)$ are related by the recursion

$\bar{\phi}_{h}(0)=\bar{\kappa}_{h}(0)=1, \sum_{i=0}^{j} \bar{\kappa}_{h}(i) \bar{\phi}_{h}(j-i)=0, j=1,2, \ldots$. 
By construction $\bar{\kappa}_{h}(z) \bar{\phi}_{h}(z)=1$ for all $|z| \leq 1$ and $\bar{\kappa}_{h}(z)$ yields the $A R(h)$ approximation to $\kappa(z)$ implicit in the bootstrap algorithm. Then $\left|\bar{\kappa}_{h}\left(e^{l \lambda}\right)\right|^{2}=$ $\left|\sum_{j=0}^{h} \bar{\phi}_{h}(j) e^{i \lambda j}\right|^{-2}=\bar{c}_{0}+\bar{c}_{1}|\lambda|^{2}+o\left(|\lambda|^{3}\right)$ as $|\lambda| \rightarrow 0$ and

$b_{T}^{*}=-\beta \frac{2 \bar{c}_{1}}{9 \bar{c}_{0}}\left(\frac{N}{T}\right)^{2}+o\left(\frac{N^{2}}{T^{2}}\right)$.

THEOREM 1. Suppose that the process y(t) satisfies Assumptions 1 and 2. Assume that $d_{T}^{f}$ is chosen such that $\left|d_{T}^{f}-d\right|<\delta_{T}$, where $\delta_{T} \log T \rightarrow 0$ as $T \rightarrow \infty$, and that an $A R(h)$ approximation is used within the prefiltered sieve bootstrap, where $h \leq H_{T}=a(\log T)^{c}, a>0, c<\infty$. Assume also that $b_{T}=E\left[\widehat{d}_{T}\right]-d$ and $b_{T}^{*}=E\left[\widehat{\vec{d}}_{T}^{* f}\right]-d_{T}^{f}$ are given by expressions (10) and (12) respectively. Then

$\left|b_{T}-b_{T}^{*}\right|=O\left(\max \left\{h\left(\frac{\log T}{T}\right)^{\frac{1}{2}-\delta_{T}}, \delta_{T} h^{-|d|}, \delta_{T} \log T\right\}\right)+o\left(\frac{N^{2}}{T^{2}}\right)$

almost surely.

It is obvious from Theorem 1 that $\left|b_{T}-b_{T}^{*}\right|=o(1)$ a.s., and not surprisingly, that the rate of convergence of $b_{T}^{*}$ to $b_{T}$ induced by the PFSB depends on the order of the autoregressive approximation $(h)$ and the proximity of the preliminary filtering value to the true $d$, that is the value of $\delta_{T}$ implicit in the choice of $d_{T}^{f}$. Which term in Theorem 1 ultimately dominates $\left|b_{T}-b_{T}^{*}\right|$, the $O\left(h(\log T / T)^{\frac{1}{2}-\delta_{T}}\right)$ or the $O\left(\delta_{T} \log T\right)$, will depend on whether $\delta_{T} \rightarrow 0$ faster or slower than $h /(T \log T)^{1 / 2}$. Given that the values of the three Landau "big-Oh"constants that appear in Theorem 1 have not been quantified, this indicates that the choice of $h$ and $d_{T}^{f}$ will have an important impact on both the finite sample and asymptotic behaviour of $b_{T}-b_{T}^{*}$. Selection of $h$ by AIC yields $h \sim K \log T$ a.s. as $T \rightarrow \infty$, which is asymptotically efficient in the sense of Shibata (1980); see Poskitt (2008) and (Politis, 2003, Section 3).

Appropriate selection of the prefiltering value for $d$ is less clear. As noted earlier, we initially choose as prefilters the actual estimators that we are interested in bias correcting, namely the LPR and SPLW estimators and their analytically biasreduced variants. Noting that in both cases the analytic bias reduction involves the inclusion of one or more even powers of frequency in the respective objective functions (see Andrews and Guggenberger, 2003 and Andrews and Sun, 2004 for details), we designate the LPR-based and SPLW-based estimators as $L P R(P)$ and $\operatorname{SPLW}(P)$ respectively, where $P=0$ indicates the original Geweke and PorterHudak (1983)/Robinson (1995a,1995b) estimators; and $P=1,2, \ldots$ indicate the corresponding bias-reduced variants based on the inclusion of $P$ even powers of the frequencies. The limiting distributions of the latter are related to those of the former via a "variance inflation factor" $\psi_{P}^{2}$; that is,

$$
N^{1 / 2}\left(\widehat{d}_{T}-d\right) \stackrel{\mathcal{D}}{\rightarrow} \mathcal{N}\left(0, \omega^{2} \psi_{P}^{2}\right)
$$


where $\omega^{2}=\pi^{2} / 24$ for $\widehat{d}_{T}$ produced via LPR, $\omega^{2}=1 / 4$ for $\widehat{d}_{T}$ produced via SPLW, $\psi_{0}^{2}=1$ yields the baseline variance for the uncorrected estimator, and $\psi_{P}^{2}$ increases with $P$. In particular, $\psi_{1}^{2}=2.25, \psi_{2}^{2}=3.52$, and $\psi_{3}^{2}=4.79$.

That each of these estimators can serve as a legitimate prefiltering value rests on the following proposition:

PROPOSITION 1. Let $d_{T}^{f}$ denote any one of the estimators $\operatorname{LPR}(P)$ or $\operatorname{SPLW}(P)$, with $P=0,1,2, \ldots$ Then under the conditions of Theorem $1 \mid d_{T}^{f}-$ $d \mid \log T \rightarrow 0$ as $T \rightarrow \infty$ with probability one.

As is made clear in Appendix A, this proposition follows directly for the $S P L W(0)$ estimator from existing results. However, for the remaining estimators detailed proofs are required. Furthermore, for a bootstrap-bias-adjusted version of an initial estimator we have $\widetilde{d}_{T}-d=\widehat{d}_{T}-d-\widehat{b}_{T, B}^{*}$, and adding and subtracting the bootstrap bias before applying the triangle inequality gives $\left|\widetilde{d}_{T}-d\right| \leq\left|\widehat{d}_{T}-d\right|+\left|\widehat{b}_{T, B}^{* f}-b_{T}^{*}\right|+\left|b_{T}^{*}\right|$. Since the bootstrap estimate of bias will obey the law of the iterated logarithm (in $B$ ) we have $\left|\widehat{b}_{T, B}^{*}-b_{T}^{*}\right|=$ $O(\sqrt{\log \log B / B})$ a.s.. Consistency and asymptotic normality of the estimator also imply that $\left|b_{T}^{*}\right|=o\left(N^{-1 / 2}\right)$. We therefore conclude that $\left|\widetilde{d}_{T}-d\right| \log T \leq$ $o(1)+\log T\left\{O(\sqrt{\log \log B / B})+o\left(N^{-1 / 2}\right)\right\} \rightarrow 0$ as $T \rightarrow \infty$ for any $B \sim K T^{\beta}$, $\beta>0$, and hence $d_{T}^{f}=\widetilde{d}_{T}$ can serve as a valid prefiltering value in a subsequent application of the algorithm. This observation prompts the extension of Section 4, in which successive bootstrap-bias-adjusted versions of the $\operatorname{LPR}(P)$ and $\operatorname{SPLW}(P)$ estimators play the role of the preliminary prefiltering value within an iterative bias-correction scheme.

\subsection{Asymptotic coverage of bootstrap confidence intervals}

The following theorem links the accuracy of the bias estimation to the accuracy with which the full sampling distribution of the relevant estimator is approximated via the bootstrap and, hence, to the coverage accuracy of the HPD confidence intervals computed using the bootstrap draws.

THEOREM 2. Set

$$
\overline{\operatorname{Pr}}^{*}\left\{N^{\frac{1}{2}}\left(\widehat{d}_{T}^{* f}-\bar{d}_{T, B}^{* f}\right)<x\right\}=B^{-1} \sum_{b=1}^{B} \mathbf{1}\left\{N^{\frac{1}{2}}\left(\widehat{d}_{T, b}^{* f}-\bar{d}_{T, B}^{* f}\right) \leq x\right\} .
$$

Then under the conditions of Theorem 1 it follows that

$$
\begin{aligned}
\sup _{x} \mid \operatorname{Pr}\left\{N^{\frac{1}{2}}\left(\widehat{d}_{T}-E\left[\widehat{d}_{T}\right]\right)<x\right\}-\overline{\operatorname{Pr}}^{*}\{ & \left.N^{\frac{1}{2}}\left(\widehat{d}_{T}^{* f}-\bar{d}_{T, B}^{* f}\right)<x\right\} \mid \\
& \leq \frac{N^{\frac{1}{2}}\left|b_{T}-b_{T}^{*}\right|}{v \sqrt{2 \pi}}+r_{B N}
\end{aligned}
$$

where the remainder $r_{B N}=N^{1 / 2} O(\sqrt{\log \log B / B})+o\left(N^{5 / 2} / T^{2}\right)$. 
Theorem 2 makes it clear that the distribution of $N^{1 / 2}\left(\widehat{d}_{T}^{* f}-E^{*}\left[\widehat{d}_{T}^{* f}\right]\right)$ will closely approximate the true finite sampling distribution of $N^{1 / 2}\left(\widehat{d}_{T}-E\left[\widehat{d}_{T}\right]\right)$ provided $N^{1 / 2}\left|b_{T}-b_{T}^{*}\right|$ is sufficiently small. Given $N=K T^{v}$ for $v \in(2 / 3,4 / 5)$, it follows from Theorem 1 that $N^{1 / 2}\left|b_{T}-b_{T}^{*}\right| \rightarrow 0$, and the accuracy with which the bootstrap-based estimate of the bias replicates the true bias as $N^{1 / 2}\left|b_{T}-b_{T}^{*}\right|$ approaches zero can be viewed as a representation of the accuracy with which the prefiltered bootstrap reproduces the true sampling distribution of the estimator per se. This implies, in turn, that for $B$ sufficiently large $\left(B \sim K T^{4 / 5+\beta}, \beta>0\right)$ HPD $\left(1-\alpha_{U}-\alpha_{L}\right) 100 \%$ confidence intervals constructed from $B$ bootstrap draws will have the correct (asymptotic) coverage. To wit, use $B$ bootstrap draws to construct the interval $\left(\widehat{d}_{T}-\widehat{q}_{T, B}^{*_{f}}\left(1-\alpha_{U}\right), \widehat{d}_{T}-\widehat{q}_{T, B}^{*_{f}}\left(\alpha_{L}\right)\right)$, with $\widehat{q}_{T, B}^{*_{f}}\left(1-\alpha_{U}\right)$ and $\widehat{q}_{T, B}^{* f}\left(\alpha_{L}\right)$ denoting the upper and lower quantiles of the narrowest interval containing $\left(1-\alpha_{U}-\alpha_{L}\right) 100 \%$ of the bootstrap distribution of the mean corrected values $\widehat{d}_{T, b}^{* f}-\bar{d}_{T, B}^{*_{f}}, b=1, \ldots, B$. Noting from (5) that $\bar{d}_{T, B}^{*_{f}}=d_{T}^{f}+\widehat{b}_{T, B}^{* f}$, we can see that the intervals so constructed correspond to bootstrap centered percentile confidence intervals that adjust for bias and accommodate possible asymmetry about the mean. ${ }^{5}$

\section{AN ITERATIVE BIAS-CORRECTION PROCEDURE}

Although the bias of $\widetilde{d}_{T}$ in (7) will be smaller than that of $\widehat{d}_{T}$, the remaining bias $E\left[\widetilde{d}_{T}\right]-d$ may still be large because the bias in any preliminary value $d_{T}^{f}$ can be severe in finite samples, and $\widehat{b}_{T, B}^{* f}$ in (5) will, as a consequence, be a biased estimate of its true counterpart $b_{T}$ in (8). To obtain a more accurate estimate of $d$ we propose a further refinement to the PFSB-based bias-correction procedure via a recursive algorithm involving two stochastic stopping criteria as follows:

1. Initialization: Set $k=0$ and assign desirable tolerance levels $\tau_{1}=\tau_{1}^{(0)}$ and $\tau_{2}=\tau_{2}^{(0)}$ for the two stopping rules. For the chosen estimator $\widehat{d}_{T}$, set $\widetilde{d}_{T}^{(0)}=\widehat{d}_{T}$ (i.e., set $d^{f}=\widehat{d}_{T}$ ).

2. Recursive Calculation: For the $k^{\text {th }}$ iteration set the preliminary value of $d$, namely $d_{T}^{f}$, to $\widetilde{d}_{T}^{(k)}$ and perform the second and third steps of the biascorrection procedure of Section 2.2 with $\widehat{d}_{T}$ therein replaced by $\widetilde{d}_{T}^{(k)}$, producing, in an obvious notation, $\widetilde{d}_{T}^{(k+1)}=\widetilde{d}_{T}^{(k)}-\widetilde{b}_{T, B}^{* f(k)}$.

3. Stopping Rules: If $\left|\widetilde{d}_{T}^{(k+1)}-\widetilde{d}_{T}^{(k)}\right|>\tau_{1}$ and $\left|\widetilde{d}_{T}^{(0)}-\widetilde{d}_{T}^{(k)}-\widetilde{b}_{T, B}^{*(k)}\right|>\tau_{2}$ set $k=k+1$, update the tolerance levels $\tau_{1}=\tau_{1}^{(k)}$ and $\tau_{2}=\tau_{2}^{(k)}$, and repeat Step 2. Otherwise set $\widetilde{d}_{T}=\widetilde{d}_{T}^{(k)}$ and stop.

The rationale behind the recursions is as follows: since the estimator $d^{f}=\widehat{d}_{T}$ is biased, $\widehat{b}_{T, B}^{* f}$ will on average be a biased estimate of $b_{T}$, and the bias-adjusted 
estimate $\widetilde{d}_{T}$ will therefore still contain some bias. Replacing the initial values $\widehat{d}_{T}=\widetilde{d}_{T}^{(0)}$ and $\widehat{b}_{T, B}^{* f}=\widetilde{b}_{T, B}^{* f}(0)$ by $\widetilde{d}_{T}^{(1)}$ and $\widetilde{b}_{T, B}^{* f(1)}$, and (for general $k$ ) $\widetilde{d}_{T}^{(k-1)}$ and $\widetilde{b}_{T, B}^{*{ }^{*}(k-1)}$ by $\widetilde{d}_{T}^{(k)}$ and $\widetilde{b}_{T, B}^{* *}$, and so on, produces more accurate estimates and bias assessments. Being based upon more accurate estimators, the updated estimate $\widetilde{d}_{T}^{(k)}$ would be expected to be closer to the true value of $d$. The procedure is iterated until no meaningful gain in accuracy is achieved. Details of the two stochastic criteria used to determine when sufficient accuracy has been attained are given in Appendix B. Some further comment on stopping rules is also included in the section following.

\section{SIMULATION EXERCISE}

\subsection{Simulation design}

In this section we illustrate the performance of the bootstrap-bias-corrected estimators via a Monte Carlo experiment. Following Andrews and Guggenberger (2003) we simulate data from an $\operatorname{ARFIMA}(1, d, 0)$ process,

$(1-L)^{d} \Phi(z) y(t)=\varepsilon(t), 0 \leq d<0.5$,

where $\Phi(z)=1-\phi z$ is the operator for a stationary $A R(1)$ component and $\varepsilon(t)$ is zero mean white noise, assumed initially to be Gaussian. The choice of this model is motivated, in part, by earlier work that highlights the distinct finite sample bias of the LPR estimator of $d$ in this setting, when the value of $\phi$ is positive and large (see Agiakloglou, Newbold, and Wohar 1993). Indeed, Andrews and Guggenberger (2003) document substantial remaining bias in the bias-corrected version of the LPR estimator in the presence of a large autoregressive parameter. The impetus for applying bootstrap-based bias correction to the various estimators is accordingly particularly strong in this setting.

The process in (14) is simulated $R=1000$ times for $d=0.0,0.2,0.3,0.4$; $\phi=0.3,0.6,0.9$; and sample sizes $T=100$ and 500 via Levinson recursion applied to the autocovariance function of the desired $\operatorname{ARFIMA}(p, d, q)$ process and the generated pseudo-random $\varepsilon(t)$ (see, for instance, Brockwell and Davis, 1991, Section 5.2). The ACF for given $T, \phi, \theta$, and $d$ is calculated using Sowell's (1992) algorithm as modified by Doornik and Ooms (2001).

The estimators to which we apply the iterative bias-correction procedure of Section 4 are $\operatorname{LPR}(P)$ and $\operatorname{SPLW}(P), P=0,1,2$, implemented with bandwidth $N=T^{0.7}$. The value of $N$ accords with common practice, with the exponent falling within the $(2 / 3,4 / 5)$ range. $^{6}$ The order $(h)$ of the autoregressive approximation underlying the sieve component of the bootstrap algorithm is chosen via AIC, and Burg's algorithm is used to estimate the autoregressive parameters. The number of bootstrap realizations is $B=1000$.

We compute the empirical bias and MSE for the original estimators prior to bootstrap-based bias correction (i.e., $\operatorname{LPR}(P)$ and $\operatorname{SPLW}(P), P=0,1,2)$, and for the bootstrap-bias-corrected versions thereof. The latter are produced through 
formal application of the stochastic stopping rules described in Appendix B, augmented by a deterministic criterion, whereby the iterative scheme ceases if $\widetilde{d}_{T}^{(k+1)}<-1$ or $\geq 1.5$ and the estimator $\widetilde{d}_{T}^{(k)}$ retained as the final choice. We also report bias and MSE results for the bootstrap-bias-corrected estimators based on the first two iterations of the iterative procedure. This comparison of the sampling properties of estimators with varying degrees of analytical bias correction with those of estimators that exploit the bootstrap bias adjustment, allows us to investigate, firstly, the efficacy of using the bootstrap method rather than an analytical method to bias adjust; and, secondly, the possibility of obtaining additional improvement by bias correcting (via the bootstrap) an estimator that has already been bias adjusted via analytical means. Finally, as a reference for the magnitude of the bias and MSE of the various raw and bias-adjusted semiparametric estimators, we record the corresponding statistics for the correctly specified MLE. ${ }^{7}$

We also compute the empirical coverage and length of nominal 95\% HPD intervals obtained by applying the bootstrap procedure to the estimators $L P R(P)$ and $\operatorname{SPLW}(P), P=0,1,2$. For each of the $R$ Monte Carlo replications the intervals are constructed as described in Section 3, each in turn based on $B$ bootstrap draws, and the empirical coverage is calculated as the proportion of times (in $R$ replications) that each interval includes the true value of $d$. The empirical length of the intervals is recorded as their average length across the $R$ replications. These coverage and length statistics are compared with the empirical coverage and (constant) length of $95 \%$ confidence intervals constructed from the appropriate asymptotic distributions in (13). Results did not vary markedly with $d$, and hence are presented averaged over the four values of $d$ considered. $^{8}$

In line with the assumption of Gaussianity, thus far we have supposed that the bootstrap innovations generated in Step 2(c) of the PFSB algorithm are i.i.d. $\mathcal{N}\left(0, \bar{\sigma}_{h}^{2}\right)$. Such bootstrap realizations are said to be generated via a parametric bootstrap. Nonparametric bootstrap innovations can be generated using the following modification of Step 2(c):

2(c's $)$ Let $\varepsilon_{h}^{+}(t), t=1, \ldots, T$, denote a simple random sample of i.i.d. values drawn from $U_{\tilde{\varepsilon}_{h}, T}(e)=T^{-1} \sum_{t=1}^{T} \mathbf{1}\left\{\tilde{\varepsilon}_{h}(t) \leq e\right\}$, the probability distribution function that places a probability mass of $1 / T$ at each of $\tilde{\varepsilon}_{h}(t)$, $t=1, \ldots, T$. Set $\varepsilon_{h}^{*}(t)=\bar{\sigma}_{h} \varepsilon_{h}^{+}(t), t=1, \ldots, T$.

The innovations generated by the nonparametric bootstrap are i.i.d. $\left(0, \bar{\sigma}_{h}^{2}\right)$ by construction, and when $y(t)$ is Gaussian we can expect $\varepsilon_{h}^{*}(t), t=1, \ldots, T$, and hence $y^{*}(t)$, to be approximately Gaussian. This suggests that replacing the innovations generated in PFSB-2(c) by those generated in $2\left(\mathrm{c}^{\prime}\right)$ should not produce outcomes that are substantially different, and we document this by presenting some selected results in which Gaussianity is retained for the data generating process, but nonparametric bootstrap innovations are generated as per $2\left(\mathrm{c}^{\prime}\right)$ above. PFSB-2( $\left.\mathrm{c}^{\prime}\right)$ also caters for the possibility that $y(t)$ is a linear process with innovations that do not satisfy Assumption 2, and so allows us to examine the robustness 
of our results to violations of the assumption of Gaussianity. Accordingly, we report some selected results obtained using the nonparametric prefiltered bootstrap with a Student $t$ distribution adopted for $\varepsilon(t)$.

Due to space considerations we tabulate in Appendix C results for the $L P R(P)$ estimators only, and for $T=500$. The $T=500$ results for the $\operatorname{SPLW}(P)$ estimators are briefly discussed in Section 5.3, but the tabulated results appear only in the extended version of the paper (http://arxiv.org/abs/1603.01897). Results (and associated discussion) for both $\operatorname{LPR}(P)$ and $\operatorname{SPLW}(P), P=0,1,2$, for $T=100$, can be found in the latter also. In all three tables included in Appendix $C$, the most favorable result (within the semiparametric set) for each design setting is highlighted. The columns headed 'SSR' in Tables 1 and 3 in Appendix C record the results based on the stochastic stopping rules discussed in Appendix B and modified as described above.

\subsection{Simulation results: LPR}

Table 1 in Appendix $\mathrm{C}$ records the bias and MSE results for all estimators based on the LPR method, using the parametric version of the bootstrap, and with Gaussian errors adopted in (14). (For brevity results for $d=0.3$ are omitted.) All results pertaining to the use of the bootstrap to bias adjust $L P R(P)$ are indicated by appending ' $-B B A(K)$ ' to the LPR acronym, where $K$ is the number of times the bootstrap-bias-correction procedure is applied. ${ }^{9}$ The key message is that the bootstrap technique does reduce bias, but with the most substantial gains to be had by using the bootstrap algorithm to bias adjust an estimator that has already been bias reduced analytically. Specifically, in seven of the nine cases, the smallest bias is produced by bias adjusting (via the bootstrap) either the $L P R(1)$ or $L P R(2)$ estimator at least once. The $L P R(2)-B B A(1)$ estimator is the least biased overall, with the reduction in bias being so substantial that this estimator also has the lowest MSE of all estimators (including those not bias adjusted) for $\phi=0.9$ and all values of $d$. Moreover, even when the bootstrap bias adjustment does cause the (expected) increase in MSE, it is not excessive. ${ }^{10}$

The results recorded in Table 1 in Appendix $\mathrm{C}$ demonstrate that using a fixed number of bootstrap-based bias adjustments is preferable overall to using an iterative (SSR) approach. With due consideration for the limitations of the experimental design, we conclude that although a stopping rule tailors the number of iterations to the realization at hand, its use does not appear to guarantee an improvement in overall performance compared to using a fixed number of iterations, at least when the sample is reasonably large. ${ }^{11}$

Note that the finding that the bias results for the bootstrap-bias-adjusted estimators are superior overall also applies to the results based on the nonparametric version of the PFSB algorithm, and under both the Gaussian and Student $t$ errors, as can be seen by the location of the highlighted figures in both panels of Table 3 in Appendix C. Again, for the purpose of brevity, results in both panels in the table are reported after averaging over all four values of $d$, including $d=0.3$. 
Another result of interest pertains to the relationship between the overall accuracy of the bootstrap-bias-corrected estimators (as measured by MSE) and that of the comparable analytically-adjusted estimators. For instance, $K$ bias adjustments of an $L P R(P)$ estimator via the bootstrap can - for some designs - yield an estimator with a smaller MSE than does the equivalent number of analytical bias adjustments. In certain cases this reduction in MSE goes hand in hand with a smaller bias. ${ }^{12}$ We return to this point in Section 5.4. With regard to overall accuracy, our results are also in accord with the findings of Nielsen and Frederiksen (2005) in that, in the absence of persistent short-run dynamics (i.e., $\phi \neq 0.9$ ), the bootstrap-bias-corrected semiparametric estimators often exhibit significant bias reduction relative to the correctly specified Gaussian MLE, with results pertaining to the latter recorded in the final column of Table 1 in Appendix C. That this improvement is at the expense of an increase in MSE relative to the MLE is perhaps not surprising given that the correctly specified MLE is (asymptotically) optimal. From a comparison of the results recorded in the final column of the two panels in Table 3 in Appendix $\mathrm{C}$ it is evident that misspecification of the innovations as Student $t$ has little impact on the performance of the Gaussian MLE. Hence, the qualitative nature of the comment made above regarding the relative performance of the semiparametric and parametric estimators continues to obtain.

Finally, the most notable characteristic of the HPD results in Table 2 in Appendix $\mathrm{C}$ (produced under the parametric algorithm) is the improvement in coverage yielded by the bootstrap technique, relative to that yielded by the relevant asymptotic distribution. In particular, use of the PFSB distributions produces intervals with close to correct coverage for the low and moderate values of $\phi$, and for the estimators based on $P \geq 1$. Unsurprisingly, while for any particular $\operatorname{LPR}(P)$ estimator (i.e., for any given value of $P$ ) the improvement in coverage accuracy is accompanied by an increase in interval width, this decrease in precision is not excessive. In both the bootstrap and asymptotic cases, an increase in $P$ tends to lead to an improvement in coverage accuracy, but at the expense (as would be expected) of an increase in interval width, due to the larger variance of the underlying estimator.

\subsection{Simulation results: SPLW}

As with the LPR-based estimators, the bootstrap-based bias adjustment is found to yield the largest bias reductions overall, but (in all but one case) only when applied to an SPLW estimator that has already been analytically bias adjusted. Most notably, the SSR method, specifically as applied to SPLW(2), yields the best bias reductions overall; although as was the case for the LPR results, it does not guarantee an improvement in performance over using a fixed number of iterations. The biases of all SPLW-based estimators are broadly similar in magnitude to those of the comparable LPR-based estimators, and as with the LPR-based estimators, the reduction in bias produced by the bootstrap technique (in certain cases) is not obtained at the expense of MSE. Once again, the qualitative results regarding 
bias adjustment still hold when the nonparametric form of the bootstrap is used, and when Student $t$ errors feature in the true DGP, rather than Gaussian errors. The MSE results demonstrate that the increase in precision sometimes yielded by the bootstrap over and above a comparable number of analytical adjustments, in the case of LPR estimator, continues to obtain in the SPLW case. As with the LPR results, the best performing bootstrap-bias-corrected SPLW-based estimators are often substantially less biased than the MLE (both correctly and incorrectly specified), for $\phi \neq 0.9$ at least, but at the expense of MSE as expected. Once again, the bootstrap yields HPD intervals with notably better coverage than those associated with the asymptotic distribution, but at some cost in precision. All supporting evidence for these statements is to be found in the relevant tables in the extended manuscript.

\subsection{Retrospective}

Our simulation results raise the question of how the sieve bootstrap as implemented in the PFSB algorithm is able to bias correct the basic LPR and SPLW estimators, and the analytically bias-adjusted versions thereof, without necessarily incurring a substantial, if any, loss in overall precision.

By way of explanation for this phenomenon, consider the LPR estimator. This estimator is commonly motivated by observing that

$$
\frac{I_{T}\left(\lambda_{j}\right) 2 \pi\left|1-e^{-l \lambda_{j}}\right|^{2 d}}{\sigma^{2}\left|\kappa\left(e^{l \lambda_{j}}\right)\right|^{2}} \stackrel{\mathcal{D}}{\rightarrow} V_{j},
$$

wherein $I_{T}(\lambda)$ denotes the periodogram and $V_{j}$ is distributed exponentially when $d=0$, and as an unequally weighted sum of independent $\chi^{2}(1)$ random variables when $d \neq 0$ (Hurvich and Beltrao, 1993, Theorem 6). Taking logarithms in (15) and using the approximation $\left|1-e^{-l \lambda}\right|^{2 d}=|\lambda|^{2 d}(1+o(1))$ as $\lambda \rightarrow 0$ leads to the linear regression model

$\log \left(I_{T}\left(\lambda_{j}\right)\right)=\alpha_{0}-2 d \log \left(\lambda_{j}\right)+\eta_{j}$,

where $E\left[\eta_{j}\right]=0$ and the intercept $\alpha_{0}$ is presumed to capture the effects of the adjustments

$$
\begin{aligned}
a_{j} & =\log |\kappa(1)|^{2}+\log \left(\frac{\left|\kappa\left(e^{\imath \lambda_{j}}\right)\right|^{2}}{|\kappa(1)|^{2}}\right)-d \log \left(\frac{\left|1-e^{-\imath \lambda_{j}}\right|^{2}}{\lambda_{j}^{2}}\right)-C_{j} \\
& =\log |\kappa(1)|^{2}-C_{j}+O\left(N^{2} / T^{2}\right) \quad \text { for all } \quad 1 \leq j \leq N,
\end{aligned}
$$

where the mean correction term $C_{j} \leq 0.577216$ (Euler's constant). ${ }^{13}$ The presumption that $\alpha_{0}$ absorbs the effects of the adjustment terms assumes the $a_{j}$ approach a constant as $T$ increases and (see the discussion in Hurvich and Beltrao, 1993) it is the failure of this assumption that can be a source of bias. 
The analytical correction replaces the simple regression in (16) by the multiple regression

$\log \left(I_{T}\left(\lambda_{j}\right)\right)=\sum_{p=0}^{P} \alpha_{p} \lambda_{j}^{2 p}-2 d \log \left(\lambda_{j}\right)+\eta_{j}$,

the rationale being that the term $\sum_{p=0}^{P} \alpha_{p} \lambda_{j}^{2 p}$ provides a better approximation to the Maclaurin series expansion of the right hand side of (17) than supposing $a_{j}$ is constant in a neighbourhood of zero. The introduction of $\lambda_{j}^{2 p}, p=1, \ldots, P$, in (19) reduces the bias in the estimate of $d$, but it is also the presence of these additional regressors that causes the variance inflation seen in (13).

The prefiltered bootstrap, on the other hand, takes the specification of the regression in (16) or (19) as given and adjusts the estimator by mimicking the sampling behaviour of the regressand. Recall that $I_{T}(\lambda)=(2 \pi)^{-1} \sum_{r=1-T}^{T-1} \widehat{\gamma}(r) e^{\imath \lambda r}$, where $\widehat{\gamma}(r)=\widehat{\gamma}(-r), r=0,1, \ldots, T-1$, denotes the sample autocovariance function. Hosking (1996) shows that even in moderate to large samples the $\widehat{\gamma}(r)$ have substantial negative bias relative to the true autocovariances when $d$ is large. The bootstrap works by reducing the value of the fractional integration parameter in the "data" to which the sieve bootstrap is applied, via the prefiltering procedure. This reduces the aforementioned bias. The reduction in $d$ also increases the proximity of the $C_{j}$ in (18) to Euler's constant and renders the $\eta_{j}$ in (16) and (19) closer to centered Gumbel random variables with variance $\pi^{2} / 6$. Whether it is applied to (16) or (19), the bootstrap is thereby able to attack the problem of bias in the estimation of $d$ without compromising (indeed, reinforcing) the fundamental result assumed to underlie log periodogram regression and determine the estimators' variance, namely, the pivotal nature of the ratio in (15).

Although the underlying reasoning is somewhat heuristic, the previous arguments provide a straightforward explanation of how the prefiltered bootstrap is able to exhibit the type of creditable performance observed in simulation when it is used to bias correct the LPR estimator. Similar arguments can also be employed to explain the performance characteristics seen when the algorithm is applied to the SPLW estimator.

\section{DISCUSSION}

This paper has developed a bootstrap method for bias correcting semiparametric estimators of the long memory parameter in fractionally integrated processes. The method involves applying the sieve bootstrap to data prefiltered by a preliminary semiparametric estimate of the long memory parameter. In addition to providing theoretical (asymptotic) justification for using the bootstrap techniques to bias correct, we document the results of simulation experiments, in which the finite sample performance of the bootstrap-bias-corrected estimators is compared with that of both unadjusted estimators and estimators adjusted via analytical means. The numerical results are very encouraging, and suggest that the bootstrap bias 
correction can yield accurate inference about long memory dynamics in the types of samples that are encountered in practice - most notably when applied to estimators for which some preliminary analytical bias reduction has been used. The bootstrap method is also shown to yield (asymptotically) valid confidence intervals that formally adjust for bias, with the empirical coverage of the bootstrap intervals being much closer to the nominal level than is the coverage of intervals based on the asymptotic distributions of the relevant semiparametric estimators.

Our discourse has focused on stationary long memory fractional processes, but as noted previously the prefiltered sieve bootstrap algorithm does not restrict either the estimator that is to be bias corrected or the prefilter to lie in the interval $[0,0.5)$. The broader applicability of the prefiltered sieve bootstrap to more general processes, and to estimators and prefilters capable of handling this generality, is therefore only contingent on establishing its theoretical validity, and as is apparent from Theorems 1 and 2 this hinges on showing that the prefiltering value $d_{T}^{f}$ is such that $\left|d_{T}^{f}-d\right| \log T=o(1)$ a.s.. If $N^{1 / 2}\left(d_{T}^{f}-d\right)$ were exactly $N(0, v)$ then it would follow from the tail area properties of the normal distribution that this condition would be satisfied. Unfortunately, approximate Gaussianity associated with a prefiltering value $d_{T}^{f}$ being a $\sqrt{N}$-CAN estimator of $d$ - as has been established for the more general estimators of Tanaka (1999) and Shimotsu and Phillips (2005) - is not sufficient to derive the required result because departures of $\left(d_{T}^{f}-d\right)$ from zero that are inconsequential for weak convergence need not be so for large-deviation probabilities. Nevertheless, the necessary large-deviation property can be derived on a case by case basis, as we have demonstrated for the LPR and SPLW estimators for the stationary case. It therefore seems reasonable to suppose that arguments similar to those employed in the proof of Proposition 1 can be used to show that the condition $\left|d_{T}^{f}-d\right| \log T=o(1)$ a.s. will also be satisfied by the aforementioned more general estimators, and under more general data generating processes, but demonstration of this is beyond the scope of the current paper. We hope to extend our results on the prefiltered sieve bootstrap to the nonstationary case in future research.

\section{NOTES}

1. The qualification 'essentially' contained in this statement refers to a deterministic stopping criterion that supplements two stochastic stopping rules applied, in turn, to the iterative version of the bias-correction method. This point is discussed further in Sections 4 and 5.1.

2. Edgeworth expansions for quadratic forms and the MLE in Gaussian long memory series are developed in Lieberman, Rousseau and Zucker (2001, 2003). See also Lieberman, Rosemarin, and Rousseau (2012).

3. Andrews, Lieberman, and Marmer (2006) examine properties of the parametric bootstrap for the current class of processes. Our aim in this exercise, however, is to avoid full parametric specifications and the associated implications of misspecification. Recent (nonbootstrap-based) work in Nadarajah, Martin, and Poskitt (2014) indicates that substantial bias can be incurred by various parametric estimators, including the Gaussian MLE, as a result of misspecification, highlighting that the nature of any misspecification would be critical to the performance of associated parametric bootstrap procedures.

4. Poskitt et al. (2015) build on Poskitt (2008) to show that under appropriate conditions, and for particular statistics, a sieve bootstrap generated sampling distribution achieves a convergence rate of 
$O_{p}\left(T^{-(1-\max \{0, d\})+\beta}\right)$ for all $\beta>0$ and $|d|<0.5$. Obviously, the closer is $d$ to zero the closer the convergence rate will be to $O_{p}\left(T^{-1+\beta}\right)$, the rate achieved with short memory (and anti-persistent) processes, and a rate arbitrarily close to that achieved with simple random samples.

5. See, inter alia, Hansen (2014, Chapter 10) and van der Vaart (1998, Chapter 23) for discussions of bootstrap confidence intervals and their associated properties.

6. A lower bound of $N \sim K T^{2 / 3}$ reflects the fact that unless $N$ increases sufficiently quickly with $T$ terms of order $O\left(\log ^{3} N / N\right)$ in the expansions of $b_{T}$ and $b_{T}^{*}$ compete with the terms in (10) and (12); and the upper bound reflects that the estimators are known to be rate optimal when $N \sim K T^{4 / 5}$ in the uncorrected case (Giraitis et al., 1997) and $N \sim K T^{(4+4 P) /(5+4 P)}$ in the corrected case (Andrews and Guggenberger, 2003; Andrews and Sun, 2004), although asymptotic normality of the estimators requires that $N=o\left(T^{4 / 5}\right)$.

7. Numerical evidence presented in Nielsen and Frederiksen (2005) suggests that semiparametric estimators can be competitive with correctly specified parametric methods. Comparison of the performance of the semiparametric estimators with that of the correctly specified, and hence asymptotically optimal, MLE is therefore of interest.

8. Analogous results for nominal $90 \%$ HPD intervals were found to be qualitatively similar in all cases and, hence, are not reported or explicitly discussed.

9. That is, $K=1$ refers to the single application of the bias-correction procedure without iteration, while $K=2$ (3, etc.) corresponds to $k=1$ (2, etc.) iterations in the iterative version of the algorithm.

10. Note that all versions of the LPR estimator, including the bootstrap-bias-corrected versions, are very biased when $\phi=0.9$. This confirms (as documented in the literature op. cit.) that semiparametric estimators experience problems in this part of the parameter space. The use of our procedure does, however, reduce the bias, indicating that even in this worst case scenario appreciable gains can be made.

11. The results recorded in the extended version of the paper for $T=100$ indicate that the stopping rules are useful for the smaller sample size, producing estimators with the smallest bias in seven cases. For $L P R(1)$, for which results for both $K=1$ and 2 are recorded, the MSE for the SSR method falls in-between the corresponding figures based on these fixed numbers of applications of the bootstrap in virtually all instances.

12. For example, for $\phi=0.3$ (and all three values of $d: 0,0.2,0.4$ ) the $L P R(1)-B B A(1)$ estimator has both bias and MSE that are less than those of the 'matching' $L P R(2)$ estimator.

13. The expression in (18) follows as a consequence of the fact that $\log \left(\left|\kappa\left(e^{l \lambda}\right)\right|^{2} /|\kappa(1)|^{2}\right)=$ $\log \left(1+\left(c_{1} / c_{0}\right)|\lambda|^{2}+o\left(|\lambda|^{3}\right)\right)$ and $\log \left(\left|1-e^{-l \lambda}\right|^{2} / \lambda^{2}\right)=\log \left(1-(1 / 12)|\lambda|^{2}+o\left(|\lambda|^{3}\right)\right)$ as $\lambda \rightarrow 0$.

\section{REFERENCES}

Agiakloglou, C., P. Newbold, \& M. Wohar (1993) Bias in the estimator of the fractional difference parameter. Journal of Time Series Analysis 14, 235-246.

Andrews, D.W., O. Lieberman, \& V. Marmer (2006) Higher-order improvements of the parametric bootstrap for long-memory Gaussian processes. Journal of Econometrics 133, 673-702.

Andrews, D.W.K. \& P. Guggenberger (2003) A bias-reduced log-periodogram regression estimator for the long-memory parameter. Econometrica 71, 675-712.

Andrews, D.W.K. \& Y. Sun (2004) Adaptive local polynomial Whittle estimation of long-range dependence. Econometrica 72, 569-614.

Apostol, T.M. (1960) Mathematical Analysis. Addison-Wesley, Reading.

Beran, J. (1994) Statistics for Long-memory Processes. Monographs on Statistics and Applied Probability, vol. 61. Chapman and Hall.

Beran, J. (1995) Maximum likelihood estimation of the differencing parameter for invertible short and long memory autoregressive integrated moving average models. Journal of the Royal Statistical Society B 57, 654-672.

Box, G. \& G. Jenkins (1970) Time Series Analysis: Forecasting and Control. Holden Day. 
Brockwell, P.J. \& R.A. Davis (1991) Time Series: Theory and Methods, 2nd ed. Springer Series in Statistics. Springer-Verlag.

Choi, E. \& P.G. Hall (2000) Bootstrap confidence regions from autoregressions of arbitrary order. Journal of the Royal Statistical Society B 62, 461-477.

Dahlhaus, R. (1989) Efficient parameter estimation for self-similar processes. Annals of Statistics 17, 1749-1766.

Doornik, J.A. \& M. Ooms (2001) Computational aspects of maximum likelihood estimation of autoregressive fractionally integrated moving average models. Computational Statistics \& Data Analysis 42, 333-348.

Doukhan, P., G. Oppenheim, \& M.S. Taqqu (eds.) (2003) Theory and Applications of Long-range Dependence. Birkhäuser Boston Inc.

Durbin, J. (1960) The fitting of time series models. Review of International Statistical Institute 28, 233-244.

Fay, G. (2010) Moment bounds for non-linear functionals of the periodogram. Stochastic Processes and their Applications 120, 983-1009.

Fay, G., E. Moulines, \& P. Soulier (2004) Edgeworth expansions for linear statistics of possibly long-range-dependent linear processes. Statistics \& Probability Letters 66, 275-288. URL http://www.sciencedirect.com/science/article/pii/S0167715203003407.

Fox, R. \& M.S. Taqqu (1986) Large sample properties of parameter estimates for strongly dependent stationary gaussian time series. Annals of Statistics 14, 517-532.

Geweke, J. \& Porter-Hudak, S. (1983) The estimation and application of long-memory time series models. Journal of Time Series Analysis 4, 221-238.

Giraitis, L. \& Robinson, P.M. (2003) Edgeworth expansions for semiparametric Whittle estimation of long memory. Annals of Statistics, 31, 1325-1375.

Giraitis, L., Robinson, P.M., \& Samarov, A. (1997) Rate optimal semiparametric estimation of the memory parameter of the gaussian time series with long-range dependence. Journal of Time Series Analysis 18, 49-60.

Granger, C.W.J. \& Joyeux, R. (1980) An introduction to long-memory time series models and fractional differencing. Journal of Time Series Analysis 1, 15-29.

Hansen, B.E. (2014) Econometrics. Online accessed 15 August 2014, URL http: //www . ssc. wisc . edu/bhansen/econometrics/.

Hosking, J.R.M. (1981) Fractional differencing. Biometrika 68, 165-176.

Hosking, J.R.M. (1996) Asymptotic distributions of the sample mean, autocovariances, and autocorrelations of long memory time series. Journal of Econometrics 73, 261-284.

Hurvich, C.M. \& K.I. Beltrao (1993) Asymptotics for the low-frequency ordinates of the periodogram of a long-memory time series. Journal of Time Series Analysis 14, 445-472.

Hurvich, C.M., R. Deo, \& J. Brodsky (1998) The mean squared error of Geweke and Porter-Hudak's estimator of the memory parameter of a long memory time series. Journal of Time Series Analysis $19,19-46$.

Inoue, A. (2002) Asymptotic behavior for partial autocorrelation functions of fractional ARIMA processes. Annals of Applied Probability 12, 1471-1491.

Inoue, A. \& Y. Kasahara (2004) Partial autocorrelation functions of the fractional ARIMA processes with negative degree of differencing. Journal of Multivariate Analysis 89, 135-147.

Inoue, A. \& Y. Kasahara (2006) Explicit representation of finite predictor coefficients and its applications. Annals of Statistics 34, 973-993.

Kreiss, J.P., E. Paparoditis, \& D.N. Politis (2011) On the range of validity of the autoregressive sieve bootstrap. Annals of Statistics 39, 2103-2130.

Levinson, N. (1947) The Wiener RMS (root mean square) error criterion in filter design and prediction. Journal of Mathematical Physics 25, 261-278.

Lieberman, O. (2001) The exact bias of the log-periodogram regression estimator. Econometric Reviews 20,369-383.

Lieberman, O., R. Rosemarin, \& J. Rousseau (2012) Asymptotic theory for maximum likelihood estimation of the memory parameter in stationary gaussian processes. Econometric Theory 28, 457-470. 
Lieberman, O., J. Rousseau, \& D.M. Zucker (2001) Valid Edgeworth expansion for the sample autocorrelation function under long range dependence. Econometric Theory 17, 257-275.

Lieberman, O., J. Rousseau, \& D.M. Zucker (2003) Valid asymptotic expansions for the maximum likelihood estimator of the parameter of a stationary, gaussian, strongly dependent process. The Annals of Statistics 31, 586-612.

Moulines, E. \& P. Soulier (1999) Broad band log-periodogram regression of time series with long range dependence. Annals of Statistics 27, 1415-1439.

Nadarajah, K., G.M. Martin, \& D.S. Poskitt (2014) Issues in the estimation of misspecified models of fractionally integrated processes. Econometrics \& Business Statistics Working Paper WP18/14, Monash University. URL http: //arxiv.org/abs/1407.1347.

Nielsen, M. \& P.H. Frederiksen (2005) Finite sample comparison of parametric, semiparametric, and wavelet estimators of fractional integration. Econometric Reviews 24, 405-443.

Politis, D.N. (2003) The impact of bootstrap methods on time series analysis. Statistical Science 18, 219-230.

Poskitt, D.S. (2007) Autoregressive approximation in nonstandard situations: The fractionally integrated and non-invertible cases. Annals of Institute of Statistical Mathematics 59, 697-725.

Poskitt, D.S. (2008) Properties of the sieve bootstrap for fractionally integrated and non-invertible processes. Journal of Time Series Analysis 29, 224-250.

Poskitt, D.S., S.D. Grose, \& G.M. Martin (2015) Higher order improvements of the sieve bootstrap for fractionally integrated processes. Journal of Econometrics 188, 94-100.

Robinson, P., (ed.) (2003) Time series with long memory. In Advanced Texts in Econometrics. Oxford University Press.

Robinson, P.M. (1995a) Gaussian semiparametric estimation of long range dependence. Annals of Statistics 23, 1630-1661.

Robinson, P.M. (1995b) Log periodogram regression of time series with long memory. Annals of Statistics 23, 1048-1072.

Shibata, R. (1980) Asymptotically efficient selection of the order of the model for estimating parameters of a linear process. Annals of Statistics 8, 147-164.

Shimotsu, K. \& P.C.B. Phillips (2005) Exact local whittle estimation of fractional integration. The Annals of Statistics 33, 1890-1933.

Sowell, F. (1992) Maximum likelihood estmation of stationary univariate fractionally integrated time series models. Journal of Econometrics 53, 165-188.

Tanaka, K. (1999) The nonstationary fractional unit root. Econometric Theory 15, 549-582.

van der Vaart, A.W. (1998) Asymptotic Statistics. Cambridge University Press.

\section{APPENDIX A. Proofs}

Proof of Theorem 1. Simple algebraic manipulation applied to (10) and (12) gives us the following bound

$$
\begin{aligned}
\left|b_{T}-b_{T}^{*}\right| & =\left|\frac{\bar{c}_{1}}{\bar{c}_{0}}-\frac{c_{1}}{c_{0}}\right| o\left(\frac{N^{2}}{T^{2}}\right)+o\left(\frac{N^{2}}{T^{2}}\right) \\
& \leq\left(\left|\frac{c_{1}\left(\bar{c}_{0}-c_{0}\right)}{c_{0} \bar{c}_{0}}\right|+\left|\frac{\left(\bar{c}_{1}-c_{1}\right)}{\bar{c}_{0}}\right|\right) o\left(\frac{N^{2}}{T^{2}}\right)+o\left(\frac{N^{2}}{T^{2}}\right) .
\end{aligned}
$$

From the first term on the right-hand-side of (A.1) it can be seen that the order of magnitude of $\left|b_{T}-b_{T}^{*}\right|$ depends on that of $\left(\bar{c}_{0}-c_{0}\right)$ and $\left(\bar{c}_{1}-c_{1}\right)$, and that the larger the bandwidth $N$, with the attendant increase in bias, the closer the approximation 
$\left|\bar{\kappa}_{h}\left(e^{l \lambda}\right)\right|^{2}$ invoked by the algorithm needs to be to the true $\left|\kappa\left(e^{l \lambda}\right)\right|^{2}$, for $\left|\bar{\kappa}_{h}\left(e^{l \lambda}\right)\right|^{2}-$ $\left|\kappa\left(e^{l \lambda}\right)\right|^{2}=\left(\bar{c}_{0}-c_{0}\right)+\left(\bar{c}_{1}-c_{1}\right)|\lambda|^{2}+o\left(|\lambda|^{3}\right)$ in a neighbourhood of the origin. Since, trivially, $2 \beta N^{2} / 9 T^{2}=O\left(N^{2} / T^{2}\right)$, in order to establish the theorem it is sufficient to show that $\left|\bar{c}_{0}-c_{0}\right|$ and $\left|\bar{c}_{1}-c_{1}\right|$ are of order $O\left(T^{2} M_{T} / N^{2}\right)$ or smaller where $M_{T}=$ $\max \left\{h\left(\frac{\log T}{T}\right)^{\frac{1}{2}-\delta_{T}}, \delta_{T} h^{-|d|}, \delta_{T} \log T\right\}$. The magnitude of both $\left(\bar{c}_{0}-c_{0}\right)$ and $\left(\bar{c}_{1}-c_{1}\right)$ can be derived from the following lemma.

LEMMA 1. Assume that the conditions of Theorem 1 hold. Let $\phi_{h}^{f}(z)=\sum_{j=0}^{h} \phi_{h}^{f}(j) z^{j}$ where $\phi_{h}^{f}(1), \ldots, \phi_{h}^{f}(h)$ denote the coefficients in the MMSEP $(h)$ of the process $w^{f}(t)$ in (4), with $d^{f}=d_{T}^{f}$, and let $\sigma_{h}^{f 2}$ denote the minimum mean squared error. Set $\kappa^{f}(z)=$ $\kappa(z) /(1-z)^{d-d_{T}^{f}}$ and define $\kappa_{h}^{f}(z)=\left\{\phi_{h}^{f}(z)\right\}^{-1}$ by replacing the coefficients of $\bar{\phi}_{h}(z)$ by those of $\phi_{h}^{f}(z)$ in the recursions in equation (11).

Then

$$
\left.\lim _{T \rightarrow \infty}|| \bar{\kappa}_{h}\left(e^{l \lambda}\right)\right|^{2}-\left|\kappa\left(e^{l \lambda}\right)\right|^{2} \mid \leq v_{1, T}+v_{2, T}+v_{3, T}
$$

where for all $\lambda \in[2 \pi / T, 2 \pi N / T]$

$$
\begin{aligned}
& v_{1, T}=\left.|| \bar{\kappa}_{h}\left(e^{\imath \lambda}\right)\right|^{2}-\left|\kappa_{h}^{f}\left(e^{\imath \lambda}\right)\right|^{2} \mid=O\left(h(\log T / T)^{\frac{1}{2}-\delta_{T}}\right) \\
& v_{2, T}=\left.|| \kappa_{h}^{f}\left(e^{l \lambda}\right)\right|^{2}-\left|\kappa^{f}\left(e^{i \lambda}\right)\right|^{2} \mid=O\left(\delta_{T} h^{-|d|}\right) \text { and } \\
& v_{3, T}=\left.|| \kappa^{f}\left(e^{l \lambda}\right)\right|^{2}-\left|\kappa\left(e^{l \lambda}\right)\right|^{2} \mid=O\left(\delta_{T} \log T\right)
\end{aligned}
$$

with probability one.

Proof of Lemma 1. Addition and subtraction, and straightforward manipulation, yields

$$
\begin{aligned}
\left|\bar{\kappa}_{h}\left(e^{l \lambda}\right)\right|^{2}-\left|\kappa\left(e^{l \lambda}\right)\right|^{2}= & \left(\left|\bar{\kappa}_{h}\left(e^{l \lambda}\right)\right|^{2}-\left|\kappa_{h}^{f}\left(e^{l \lambda}\right)\right|^{2}\right)+\left(\left|\kappa_{h}^{f}\left(e^{l \lambda}\right)\right|^{2}-\left|\kappa^{f}\left(e^{l \lambda}\right)\right|^{2}\right) \\
& +\left(\left|\kappa^{f}\left(e^{l \lambda}\right)\right|^{2}-\left|\kappa\left(e^{l \lambda}\right)\right|^{2}\right) .
\end{aligned}
$$

Consider the first term in (A.2), $\left|\bar{\kappa}_{h}\left(e^{\imath \lambda}\right)\right|^{2}-\left|\kappa_{h}^{f}\left(e^{\imath \lambda}\right)\right|^{2}$. By definition

$\bar{\kappa}_{h}(z)-\kappa_{h}^{f}(z)=\frac{\phi_{h}^{f}(z)-\bar{\phi}_{h}(z)}{\bar{\phi}_{h}(z) \phi_{h}^{f}(z)}$,

and since $\bar{\phi}_{h}(z) \neq 0$ and $\phi_{h}^{f}(z) \neq 0,|z| \leq 1$, there exists an $\epsilon>0$ such that

$$
\begin{aligned}
\left|\bar{\kappa}_{h}(z)-\kappa_{h}^{f}(z)\right| & \leq \epsilon^{-2}\left|\phi_{h}^{f}(z)-\bar{\phi}_{h}(z)\right| \\
& \leq \epsilon^{-2} \sum_{j=0}^{h}\left|\phi_{h}^{f}(j)-\bar{\phi}_{h}(j)\right| \text { for all }|z| \leq 1 .
\end{aligned}
$$


But

$$
\begin{aligned}
\sum_{j=0}^{h}\left|\phi_{h}^{f}(j)-\bar{\phi}_{h}(j)\right| & \leq\left(h \sum_{j=0}^{h}\left|\phi_{h}^{f}(j)-\bar{\phi}_{h}(j)\right|^{2}\right)^{\frac{1}{2}} \\
& =O\left(h\left(\frac{\log T}{T}\right)^{\frac{1}{2}\left(1-2 \max \left\{0, d-d_{T}^{f}\right\}\right)}\right) \\
& =O\left(h\left(\frac{\log T}{T}\right)^{\frac{1}{2}-\delta_{T}}\right) \text { a.s. }
\end{aligned}
$$

by an application of Poskitt (2007, Theorem 5) and the fact that $\left|d_{T}^{f}-d\right|<\delta_{T}$ by assumption. It follows that $\left|\bar{\kappa}_{h}\left(e^{l \lambda}\right)-\kappa_{h}^{f}\left(e^{l \lambda}\right)\right|=O\left(h(\log T / T)^{\frac{1}{2}-\delta_{T}}\right)$ a.s. uniformly in $\lambda$, and hence that $\left.|| \bar{\kappa}_{h}\left(e^{l \lambda}\right)\right|^{2}-\left|\kappa_{h}^{f}\left(e^{l \lambda}\right)\right|^{2} \mid=O\left(h(\log T / T)^{\frac{1}{2}-\delta_{T}}\right)$ a.s. uniformly in $\lambda$. We can therefore interchange limit operations (Apostol, 1960, Theorem 13.3) to give

$\left.\lim _{T \rightarrow \infty \lambda \rightarrow 0} \lim _{\lambda \rightarrow 0}|| \bar{\kappa}_{h}\left(e^{i \lambda}\right)\right|^{2}-\left|\kappa_{h}^{f}\left(e^{i \lambda}\right)\right|^{2}\left|=\lim _{\lambda \rightarrow 0} \lim _{T \rightarrow \infty}\right|\left|\bar{\kappa}_{h}\left(e^{l \lambda}\right)\right|^{2}-\left|\kappa_{h}^{f}\left(e^{l \lambda}\right)\right|^{2} \mid$,

which implies that $v_{1, T}=O\left(h(\log T / T)^{\frac{1}{2}-\delta_{T}}\right)$ a.s. for all $\lambda \in[2 \pi / T, 2 \pi N / T]$.

For the second term in (A.2), $\left|\kappa_{h}^{f}\left(e^{l \lambda}\right)\right|^{2}-\left|\kappa^{f}\left(e^{l \lambda}\right)\right|^{2}$, we have

$\kappa_{h}^{f}(z)-\kappa^{f}(z)=\frac{1-\kappa^{f}(z) \phi_{h}^{f}(z)}{\phi_{h}^{f}(z)}$,

giving us the bound

$\left|\kappa_{h}^{f}(z)-\kappa^{f}(z)\right| \leq \epsilon^{-1}\left|1-\kappa^{f}(z) \phi_{h}^{f}(z)\right| \quad$ for all $|z| \leq 1$.

Let $\rho_{h}(z)=\sum_{j \geq 1} \rho_{h}(j) z^{j}=1-\kappa^{f}(z) \phi_{h}^{f}(z)$. Then from Parseval's relation

$\sum_{j \geq 1} \rho_{h}(j)^{2}=\int_{-\pi}^{\pi}\left|1-\kappa^{f}\left(e^{l \lambda}\right) \phi_{h}^{f}\left(e^{l \lambda}\right)\right|^{2} d \lambda=2 \pi \sigma^{-2}\left(\sigma_{h}^{f 2}-\sigma^{2}\right)$

and from the Levinson-Durbin recursions (Levinson, 1947; Durbin, 1960) we have $\sigma_{h}^{f 2}=\left(1-\phi_{h}^{f}(h)^{2}\right) \sigma_{h-1}^{f 2}$. Substituting sequentially in the recurrence formula $\sigma_{h}^{f 2}=$ $\sigma_{h+1}^{f 2}+\phi_{h}^{f}(h)^{2} \sigma_{h}^{f 2}$ leads to the series expansion $\sigma_{h}^{f 2}-\sigma^{2}=\sum_{r=h}^{\infty} \phi_{r}^{f}(r)^{2} \sigma_{r}^{f 2}$, from which we obtain the bound

$\sum_{j \geq 1} \rho_{h}(j)^{2} \leq 2 \pi \sigma^{-2} E\left[w^{f}(t)^{2}\right] \sum_{r=h}^{\infty} \phi_{r}^{f}(r)^{2}$.

But $\phi_{h}^{f}(h) \sim\left|d_{T}^{f}-d\right| / h$ as $h \rightarrow \infty$ (Inoue, 2002; Inoue and Kasahara, 2004) and therefore we can infer that

$\sum_{j \geq 1} \rho_{h}(j)^{2} \leq \mathrm{const} \cdot \frac{\left|d_{T}^{f}-d\right|^{2}}{h^{2|d|}} \zeta(2(1-|d|))$, 
where $\zeta(\cdot)$ denotes the Riemann zeta function. It follows that $\lim _{h \rightarrow \infty} \rho_{h}\left(e^{l \lambda}\right)=0$ and that $\lim _{T \rightarrow \infty}\left|\rho_{h}\left(e^{l \lambda}\right)\right|^{2}=O\left(\delta_{T}^{2} h^{-2|d|}\right)$ almost everywhere on $[-\pi, \pi]$. Hence we can conclude that $\left.\lim _{T \rightarrow \infty} \lim _{\lambda \rightarrow 0}|| \kappa_{h}^{f}\left(e^{i \lambda}\right)\right|^{2}-\left|\kappa^{f}\left(e^{i \lambda}\right)\right|^{2} \mid=$ $\left.\lim _{\lambda \rightarrow 0} \lim _{T \rightarrow \infty}|| \kappa_{h}^{f}\left(e^{l \lambda}\right)\right|^{2}-\left|\kappa^{f}\left(e^{l \lambda}\right)\right|^{2} \mid$ and hence that $\nu_{2, T}=O\left(\delta_{T}^{2} h^{-2|d|}\right)$.

The third and final term in (A.2) is

$$
\left|\kappa^{f}\left(e^{i \lambda}\right)\right|^{2}-\left|\kappa\left(e^{l \lambda}\right)\right|^{2}=\left|\kappa\left(e^{l \lambda}\right)\right|^{2}\left(\left|1-e^{l \lambda}\right|^{2\left(d_{T}^{f}-d\right)}-1\right) .
$$

Substituting $\left|1-e^{\imath \lambda}\right|^{2\left(d_{T}^{f}-d\right)}=\exp \left\{\left(d_{T}^{f}-d\right) \log \left|1-e^{\imath \lambda}\right|^{2}\right\}$ into (A.3) and using the expansion $\left|1-e^{-\imath \lambda}\right|^{2}=2 \sum_{j=1}^{\infty}(-1)^{j-1}|\lambda|^{2 j} /(2 j)$ !, which implies that $\log \left|1-e^{\imath \lambda}\right|^{2}=$ $2 \log |\lambda|+\log (1+o(|\lambda|))$ as $\lambda \rightarrow 0$, we can deduce that

$$
\left.|| \kappa\left(e^{\imath \lambda}\right)\right|^{2}\left(\left|1-e^{\imath \lambda}\right|^{2\left(d_{T}^{f}-d\right)}-1\right)\left|\leq\left\{\sup _{[-\pi, \pi]}\left|\kappa\left(e^{\imath \lambda}\right)\right|^{2}\right\}\right| \exp \left\{2\left(d_{T}^{f}-d\right) \log |\lambda|+o(|\lambda|)\right\}-1 \mid
$$

as $\lambda \rightarrow 0$. Furthermore, by assumption $\left|d_{T}^{f}-d\right| \leq \delta_{T}$ where $\delta_{T} \log T \rightarrow 0$ as $T \rightarrow \infty$, and since $|\exp (x)-1|=|x| \cdot\left|1+\frac{1}{2} x+o(|x|)\right|$ for $x$ in a neighbourhood of the origin, it follows that

$$
\left.|| \kappa\left(e^{l \lambda}\right)\right|^{2}\left(\left|1-e^{l \lambda}\right|^{2\left(d_{T}^{f}-d\right)}-1\right)\left|\leq 2\left\{\sup _{[-\pi, \pi]}\left|\kappa\left(e^{\imath \lambda}\right)\right|^{2}\right\}\right| d_{T}^{f}-d||(\log 2 \pi N / T)+o(N / T) \mid
$$

for all $\lambda \in[2 \pi / T, 2 \pi N / T]$ as $T \rightarrow \infty$. We can therefore infer that (A.3) is $O\left(\delta_{T} \log T\right)$ or smaller, uniformly in $\lambda$ for all $\lambda \in[2 \pi / T, 2 \pi N / T]$. This completes the proof of Lemma 1.

Returning to the proof of Theorem 1, evaluating the expression

$$
\left(\bar{c}_{0}-c_{0}\right)+\left(\bar{c}_{1}-c_{1}\right)|\lambda|^{2}=\left|\bar{\kappa}_{h}\left(e^{l \lambda}\right)\right|^{2}-\left|\kappa\left(e^{l \lambda}\right)\right|^{2}+o\left(|\lambda|^{3}\right)
$$

at $\lambda=2 \pi / T$ and $2 \pi N / T$, and solving for $\bar{c}_{0}-c_{0}$ and $\bar{c}_{1}-c_{1}$, it follows as a consequence of Lemma 1 that $\left|\bar{c}_{0}-c_{0}\right|=O\left(M_{T}\right)+o\left(T^{-3}\right)$ and $\left|\bar{c}_{1}-c_{1}\right|=O\left(T^{2} M_{T} / N^{2}\right)+o(N / T)$. Extracting the dominant term completes the proof of Theorem 1.

Proof of Proposition 1. Let $\widehat{d}_{T}$ denote the $\operatorname{LPR}(0)$ estimator. Then $\widehat{d}_{T}$ is the OLS coefficient of the regressor $-2 \log \lambda_{j}$ in the regression of $\log I_{T}\left(\lambda_{j}\right)$ on 1 and $-2 \log \lambda_{j}$. Substituting $a_{j}-2 d \log \left(\lambda_{j}\right)+\eta_{j}$ for $\log I_{T}\left(\lambda_{j}\right)$ in this regression leads to the expression

$$
\begin{aligned}
\widehat{d}_{T}-d & =-\frac{\sum_{j=1}^{N}\left(\log \lambda_{j}-\overline{\log \lambda}\right)\left(\eta_{j}+a_{j}\right)}{2 \sum_{j=1}^{N}\left(\log \lambda_{j}-\overline{\log \lambda}\right)^{2}} \\
& =-\frac{1}{2} \sum_{j=1}^{N} r_{j}\left(\eta_{j}+a_{j}\right)
\end{aligned}
$$


for the estimation error where $\eta_{j}$ and $a_{j}$ are defined in expressions (16) and (17), and $r_{j}=\left(\log \lambda_{j}-\overline{\log \lambda}\right) / \sum_{j=1}^{N}\left(\log \lambda_{j}-\overline{\log \lambda}\right)^{2}, j=1, \ldots, N$. See the discussion associated with (16) and (17) for clarification.

By Theorem 2 of Moulines and Soulier (1999) there exist sequences $e_{j}$ and $f_{j}$, $j=1, \ldots, N$, such that $\eta_{j}=e_{j}+f_{j}$, where the $e_{j}, j=1, \ldots, N$, are weakly dependent, centered Gumbel random variables with variance $\pi^{2} / 6$ and covariance $\operatorname{cov}\left\{e_{k}, e_{j}\right\}=$ $O\left(\log ^{2}(j) k^{-2|d|} j^{2(|d|-1)}\right)$ for $1 \leq k<j \leq N$, and $\left|f_{j}\right|=O(\log (1+j) / j)$ with probability one. Since $\max _{1 \leq j \leq N}\left|\log \lambda_{j}-\overline{\log \lambda}\right|=O(\log N)$ and $\sum_{j=1}^{N}\left(\log \lambda_{j}-\overline{\log \lambda}\right)^{2}=O(N)$ it follows that $\sum_{j=1}^{N} r_{j} f_{j}=O\left(\log ^{3} N / N\right)$ a.s.. Given that $\sum_{j=1}^{N} r_{j}=0$, it also follows from (18) that $\sum_{j=1}^{N} r_{j} a_{j}=O\left(N^{2} \log N / T^{2}\right)$. We can therefore infer from (A.5) that

$\widehat{d}_{T}-d=-\frac{1}{2} \sum_{j=1}^{N} r_{j} e_{j}+R_{N}$

where $\left|R_{N}\right| \log T=O\left(v^{3} \log ^{4} T / T^{v}\right)+O\left(v \log ^{2} T / T^{2(1-v)}\right)=o(1)$ a.s., $2 / 3<v<4 / 5$.

The desired result now follows because on application of a law of large numbers for triangular arrays of weakly dependent random variables we find that for all $\delta>0$

$\sum_{j=1}^{N} r_{j} e_{j}=o\left((v \log T)^{5 / 2}(\log (v \log T))^{(1+\delta) / 2} T^{-v / 2}\right) \quad$ a.s..

More specifically, let $S_{n}=\sum_{j=1}^{n} r_{j} e_{j}$. Then by Doob's inequality $E\left[\left(\max _{n \leq 2^{k}}\left|S_{n}\right|\right)^{2}\right] \leq$ $4 E\left[\left|S_{2^{k}}\right|^{2}\right]$, and using the bounds on the covariance of $e_{j}$ we have

$E\left[\left|S_{n}\right|^{2}\right]=\sum_{j=1}^{n} r_{j}^{2} E\left[e_{j}^{2}\right]+2 \sum_{1 \leq k<j \leq n} r_{k} r_{j} \operatorname{cov}\left\{e_{k}, e_{j}\right\}=O\left(\log ^{4} n / n\right)$.

We can therefore conclude that for any $\delta>0$

$\sum_{k=1}^{\infty} \frac{2^{k}}{k^{5}(\log k)^{1+\delta}} E\left[\left(\max _{n \leq 2^{k}}\left|S_{n}\right|\right)^{2}\right] \leq \sum_{k=1}^{\infty} \frac{2^{k}}{k^{5}(\log k)^{1+\delta}} O\left(\frac{k^{4}}{2^{k}}\right)<\infty$,

since $\sum_{k=1}^{\infty} 1 / k(\log k)^{1+\delta}<\infty$, which by the Borel-Cantelli lemma implies $\max _{n \leq 2^{k}}\left|S_{n}\right|=o\left(k^{5 / 2}(\log k)^{(1+\delta) / 2} 2^{-k / 2}\right) \quad$ a.s.. $\quad$ Consequently $\quad \sqrt{N}\left|S_{N}\right|=$ $o\left((\log N)^{5 / 2}(\log \log N)^{(1+\delta) / 2}\right)$ a.s. since the function $(\log n)^{5 / 2}(\log \log n)^{(1+\delta) / 2}$ is slowly varying at infinity.

Now let $\widehat{d}_{T}$ denote the $\operatorname{LPR}(P)$ estimator with $P \geq 1$. The analytically-bias-adjusted LPR estimator is the OLS coefficient of the regressor $-2 \log \lambda_{j}$ in the regression of $\log I_{T}\left(\lambda_{j}\right)$ on $1,-2 \log \lambda_{j}$, and $\lambda_{j}^{2 p}, p=1, \ldots, P$. Applying the Frisch-Waugh-Lovell theorem and projecting out the regressors $\lambda_{j}^{2 p}, p=1, \ldots, P$, as well as unity we can express the estimation error $\widehat{d}_{T}-d$ exactly as in (A.5), save that the $r_{j}$ are now defined in terms of $-2 \widetilde{\log \lambda_{j}}$, say, the component of $-2 \log \lambda_{j}$ orthogonal to 1 and $\lambda_{j}^{2 p}, p=1, \ldots, P$. 
This projection does not alter the overall magnitudes, so for the orthogonalized regressor we have $\max _{1 \leq j \leq N}\left|\widetilde{\log \lambda_{j}}\right|=O(\log N)$ and $\sum_{j=1}^{N}\left(\widetilde{\log \lambda_{j}}\right)^{2}=O(N)$ (Andrews and Guggenberger, 2003, Lemma 2, parts (j) \& (k)). The proof that $\left|\widehat{d}_{T}-d\right| \log T=o$ (1) a.s. now proceeds as previously with $r_{j}=\widetilde{\log \lambda_{j}} / \sum_{j=1}^{N}\left(\log \lambda_{j}\right)^{2}, j=1, \ldots, N$.

For the $S P L W(0)$ estimator the proposition follows directly from Giraitis and Robinson (2003, Lemma 5.8), which implies that the SPLW estimator satisfies $P\left(\left|\widehat{d}_{T}-d\right| \log T>\right.$ $\epsilon)=o\left(N^{-p}\right)$, where $p>1 / \epsilon$ and $N$, the bandwidth, satisfies $T^{\epsilon}<N<T^{1-\epsilon}$ for some $\epsilon>0$. For the $\operatorname{SPLW}(P \geq 1)$ estimator the proposition can be established in a manner similar to that employed above for the $L P R(P)$ estimators. Using Lemma 4 of Andrews and Sun (2004) we can express $\widehat{d}_{T}-d$, where $\widehat{d}_{T}$ now denotes the $\operatorname{SPLW}(P)$ estimator with $P \geq 1$, as a function of the standardized score and from Andrews and Sun (2004, Lemma 5) we can conclude that the standardized score is of an order that implies that $\left|\widehat{d}_{T}-d\right| \log T=o(1) a . s . ; c f$. Andrews and Sun (2004, Theorem 4).

Proof of Theorem 2. The sampling distribution of $N^{1 / 2}\left(\widehat{d}_{T}-d\right)$ for the LPR and SPLW estimators, under the Gaussianity assumption, admits a normal approximation such that

$$
\sup _{x}\left|\operatorname{Pr}\left\{\frac{N^{1 / 2}\left(\widehat{d}_{T}-d\right)}{v}<x\right\}-G(x)\right|=o\left(\frac{N^{5 / 2}}{T^{2}}\right),
$$

where $v=\omega \psi_{P}$ and $G(\cdot)$ denotes the standard normal distribution function. Substituting (8) into (A.6) gives the approximation

$$
\begin{aligned}
\operatorname{Pr}\left\{N^{1 / 2}\left(\widehat{d}_{T}-E\left[\widehat{d}_{T}\right]\right)<x\right\} & =\operatorname{Pr}\left\{N^{1 / 2}\left(\widehat{d}_{T}-d\right)<x+N^{1 / 2} b_{T}\right\} \\
& =G\left(\left(x+N^{1 / 2} b_{T}\right) / v\right)+o\left(N^{5 / 2} / T^{2}\right)
\end{aligned}
$$

for the distribution of the finite sample deviation $\widehat{d}_{T}-E\left[\widehat{d}_{T}\right]$. Similarly,

$$
\sup _{x}\left|\operatorname{Pr}^{*}\left\{\frac{N^{1 / 2}\left(\widehat{d}_{T}^{* f}-d_{T}^{f}\right)}{v}<x\right\}-G(x)\right|=o\left(\frac{N^{5 / 2}}{T^{2}}\right),
$$

and substituting (9) into (A.8) we obtain the approximation

$$
\begin{aligned}
\operatorname{Pr}^{*}\left\{N^{1 / 2}\left(\widehat{d}_{T}^{* f}-E^{*}\left[\widehat{d}_{T}^{* f}\right]\right)<x\right\} & =\operatorname{Pr}^{*}\left\{N^{1 / 2}\left(\widehat{d}_{T}^{* f}-d_{T}^{f}\right)<x+N^{1 / 2} b_{T}^{*}\right\} \\
& =G\left(\left(x+N^{1 / 2} b_{T}^{*}\right) / v\right)+o\left(N^{5 / 2} / T^{2}\right)
\end{aligned}
$$

for the bootstrap deviation $\widehat{d}_{T}^{*_{f}}-E^{*}\left[\widehat{d}_{T}^{* f}\right]$. Subtracting (A.7) from (A.9) we find that the difference between $\operatorname{Pr}^{*}\left\{N^{1 / 2}\left(\widehat{d}_{T}^{* f}-E^{*}\left[\widehat{d}_{T}^{* f}\right]\right)<x\right\}$ and $\operatorname{Pr}\left\{N^{1 / 2}\left(\widehat{d}_{T}-E\left[\widehat{d}_{T}\right]\right)<x\right\}$ is bounded in absolute value by

$$
\left|G\left(\left(x+N^{1 / 2} b_{T}\right) / v\right)-G\left(\left(x+N^{1 / 2} b_{T}^{*}\right) / v\right)\right|+o\left(N^{5 / 2} / T^{2}\right),
$$

and

$$
\sup _{x}\left|G\left(\left(x+N^{1 / 2} b_{T}\right) / v\right)-G\left(\left(x+N^{1 / 2} b_{T}^{*}\right) / v\right)\right| \leq \frac{N^{1 / 2}}{v \sqrt{2 \pi}}\left|b_{T}-b_{T}^{*}\right|
$$


by the first mean value theorem for integrals (Apostol, 1960, Theorem 7.30).

Recognizing that $\bar{d}_{T, B}^{* f}=d_{T}^{f}+\widehat{b}_{T, B}^{* f}$ and employing the expansion (A.8) once more we also have the approximation

$$
\begin{aligned}
\operatorname{Pr}^{*}\left\{N^{\frac{1}{2}}\left(\widehat{d}_{T}^{* f}-\bar{d}_{T, B}^{* f}\right)<x\right\} & =\operatorname{Pr}^{*}\left\{N^{\frac{1}{2}}\left(\widehat{d}_{T}^{* f}-d_{T}^{f}\right)<x+N^{\frac{1}{2}} \widehat{b}_{T, B}^{* f}\right\} \\
& =G\left(\left(x+N^{\frac{1}{2} \widehat{b}_{T, B}^{* f}}\right) / v\right)+o\left(N^{5 / 2} / T^{2}\right) .
\end{aligned}
$$

Subtracting the approximation in (A.10) from that in (A.7) and using the triangle inequality, having added and subtracted (A.9), now yields the result that

$$
\sup _{x}\left|\operatorname{Pr}\left\{N^{\frac{1}{2}}\left(\widehat{d}_{T}-E\left[\widehat{d}_{T}\right]\right)<x\right\}-\operatorname{Pr}^{*}\left\{N^{\frac{1}{2}}\left(\widehat{d}_{T}^{* f}-\bar{d}_{T, B}^{* f}\right)<x\right\}\right| \leq \frac{N^{\frac{1}{2}}\left|b_{T}-b_{T}^{*}\right|}{v \sqrt{2 \pi}}+r_{B N},
$$

where the remainder $r_{B N}=N^{\frac{1}{2}} O(\sqrt{\log \log B / B})+o\left(N^{5 / 2} / T^{2}\right)$. The first term on the right hand side in (A.11) follows from the inequality $\sup _{x} \mid G\left(\left(x+N^{\frac{1}{2}} b_{T}\right) / v\right)-G((x+$ $\left.\left.N^{\frac{1}{2}} b_{T}^{*}\right) / v\right)\left|\leq N^{\frac{1}{2}}\right| b_{T}-b_{T}^{*} \mid / v \sqrt{2 \pi}$, and similarly, the first term of the remainder derives from the inequality $\sup _{x}\left|G\left(\left(x+N^{\frac{1}{2}} \widehat{b}_{T, B}^{*_{f}}\right) / v\right)-G\left(\left(x+N^{\frac{1}{2}} b_{T}^{*}\right) / v\right)\right| \leq N^{\frac{1}{2}} \mid \widehat{b}_{T, B}^{*_{f}}-$ $b_{T}^{*} \mid / v \sqrt{2 \pi}$, since $\left|\widehat{b}_{T, B}^{*}-b_{T}^{*}\right|=O(\sqrt{\log \log B / B})$ a.s.. Furthermore, if we set

$\overline{\operatorname{Pr}}^{*}\left\{N^{\frac{1}{2}}\left(\widehat{d}_{T}^{* f}-\bar{d}_{T, B}^{* f}\right)<x\right\}=B^{-1} \sum_{b=1}^{B} \mathbf{1}\left\{N^{\frac{1}{2}}\left(\widehat{d}_{T, b}^{* f}-\bar{d}_{T, B}^{* f}\right) \leq x\right\}$

then by the Dvoretsky-Kiefer-Wolfowitz inequality the probability of the event $\sup _{x}\left|\overline{\operatorname{Pr}}^{*}\left\{N^{\frac{1}{2}}\left(\widehat{d}_{T}^{* f}-\bar{d}_{T, B}^{* f}\right)<x\right\}-\operatorname{Pr}^{*}\left\{N^{\frac{1}{2}}\left(\widehat{d}_{T}^{* f}-\bar{d}_{T, B}^{*_{f}}\right)<x\right\}\right|>\delta$

is bounded by $2 \exp \left(-2 B \delta^{2}\right)$. It therefore follows that for all $B \sim K T^{4 / 5+\beta}, \beta>0$, the $\left(1-\alpha_{U}-\alpha_{L}\right) 100 \%$ significance level HPD intervals are consistent with respect to the Kolmogorov-Smirnov metric.

\section{APPENDIX B. Stochastic Stopping Rules}

Two criteria are used to determine if any meaningful gain in accuracy will be achieved by adding a further iteration to the iterative procedure of Section 4 . The first, $\left|\widetilde{d}_{T}^{(k+1)}-\widetilde{d}_{T}^{(k)}\right|>$ $\tau_{1}^{(k)}$, is based on Cauchy's convergence criterion. Given the stochastic nature of the bias correction mechanism we can think of this as a statistical decision rule in which $\tau_{1}^{(k)}$ governs the probability of moving from the $k^{t h}$ to the $(k+1)^{t h}$ iteration. Now $\tilde{d}_{T}^{(k+1)}-\widetilde{d}_{T}^{(k)}=-\widetilde{b}_{T, B}^{*_{f}(k)}=-\frac{1}{B} \sum_{b=1}^{B}\left(\tilde{d}_{T, b}^{*_{f}(k)}-\widetilde{d}_{T}^{(k)}\right)$, where $\tilde{d}_{T, b}^{*_{f}(k)}$ denotes the estimator produced from the $b^{t h}$ PFSB draw with $\widetilde{d}_{T}^{(k)}$ used as the prefiltering value; and 
since $\widehat{d}_{T}$ is a $\sqrt{N}-\mathrm{CAN}$ estimator, given the data and the current and previous bootstrap iterations, $N^{\frac{1}{2}}\left(\tilde{d}_{T, b}^{* f}(k)-\tilde{d}_{T}^{(k)}\right) \stackrel{\mathcal{D}}{\rightarrow} \mathcal{N}\left(0, v^{2}\right)$. The conditional (asymptotic) variance of $B^{-1} \sum_{b=1}^{B}\left(\tilde{d}_{T, b}^{* f}(k)-\widetilde{d}_{T}^{(k)}\right)$ is therefore $v^{2} / N B$, and using the rule that the overall variance equals the variance of the conditional mean (in this case $\operatorname{Var}\left[\widetilde{d}_{T}^{(k)}\right]$ ) plus the expectation of the conditional variance (in this case the constant $v^{2} / N B$ ) we can infer that the (asymptotic) variance of the difference between successive bias-adjusted estimators is given by $\operatorname{Var}\left[\widetilde{d}_{T}^{(k+1)}-\widetilde{d}_{T}^{(k)}\right]=\operatorname{Var}\left[\widetilde{d}_{T}^{(k)}\right]+\frac{v^{2}}{N B}$. Furthermore, from the recurrence formula $\widetilde{d}_{T}^{(k)}=\widetilde{d}_{T}^{(k-1)}-\widetilde{b}_{T, B}^{*_{f}(k-1)}=\widetilde{d}_{T}^{(k-1)}-\frac{1}{B} \sum_{b=1}^{B}\left(\tilde{d}_{T, b}^{*_{f}(k-1)}-\widetilde{d}_{T}^{(k-1)}\right)$, it follows by a similar logic that $\operatorname{Var}\left[\widetilde{d}_{T}^{(k)}\right]=2 \cdot \operatorname{Var}\left[\widetilde{d}_{T}^{(k-1)}\right]+\frac{v^{2}}{N B}$, where $\operatorname{Var}\left[\widetilde{d}_{T}^{(1)}\right]=$ $2 \cdot \operatorname{Var}\left[\widetilde{d}_{T}^{(0)}\right]+v^{2} / N B=(2 B+1) v^{2} / N B$. Moreover, at each iteration the bias-adjusted estimate is constructed as a linear combination of asymptotically normal random variables and is itself therefore asymptotically normal. This indicates that $\tau_{1}^{(k)}$ can be evaluated from percentile points of the normal approximation.

Similarly, the second convergence criterion, $\left|\widetilde{d}_{T}^{(0)}-\widetilde{d}_{T}^{(k)}-\widetilde{b}_{T, B}^{*_{f}(k)}\right|>\tau_{2}^{(k)}$, is perhaps best thought of as the decision rule that examines the difference between the current accumulated bias correction, $\widetilde{d}_{T}^{(0)}-\widetilde{d}_{T}^{(k)}$, and the current bootstrap estimate of the bias, $\widetilde{b}_{T, B}^{*_{f}(k)}$. From the expression $\widetilde{d}_{T}^{(0)}-\widetilde{d}_{T}^{(k)}-\widetilde{b}_{T, B}^{*_{f}(k)}=\widetilde{d}_{T}^{(0)}-\left(\frac{1}{B} \sum_{b=1}^{B} \tilde{d}_{T, b}^{*_{f}(k)}\right)$, it follows that the (asymptotic) variance, $\operatorname{Var}\left[\widetilde{d}_{T}^{(0)}-\widetilde{d}_{T}^{(k)}-\widetilde{b}_{T, B}^{* f}(k)\right]=\frac{v^{2}}{N}\left(1+2^{k-1}\left[1+\frac{1}{B}\right]\right)$, and the tolerance level $\tau_{2}^{(k)}$ can once again be set using percentile points from the asymptotic normal approximation.

The interpretation of the convergence criteria as statistical decision rules in which the tolerance levels govern the probability of going from the current to the next iteration suggests that $\tau_{1}^{(k)}$ and $\tau_{2}^{(k)}$ be set by reference to conventional critical values used in statistical hypothesis tests. When $k$ is very small we might conjecture that $\widetilde{d}_{T}^{(k)}$ still contains some bias and we may wish to iterate further unless there is strong evidence that so doing will produce very little change. On the other hand, when $k$ is large the initial estimate $\widetilde{d}_{T}^{(0)}$ has already undergone several adjustments to produce $\widetilde{d}_{T}^{(k)}$ and we may prefer to terminate iteration unless there is strong evidence that further iteration will produce additional, substantial correction. We can therefore calibrate $\tau_{1}^{(k)}$ and $\tau_{2}^{(k)}$ using quantile points of the normal distribution $z_{\left(1-p_{k} / 2\right)}$ (where $G\left(z_{(1-p)}\right)=1-p$ ) and $p_{k}$, the probability of going from the $k^{t h}$ to the $(k+1)^{t h}$ iteration, is assigned to be large when $k$ is small and vice versa. In the simulation experiments we set $p_{0}=0.95, p_{1}=0.9$, and $p_{k}=(0.1) 2^{(1-k)}$ for $k=2,3, \ldots$ for $\operatorname{LPR}(0)$ and $\operatorname{SPLW}(0)$; and $p_{0}=0.9, p_{k}=(0.1) 2^{-k}$ for $k=1,2,3, \ldots$ for $\operatorname{LPR}(P)$ and $\operatorname{SPLW}(P), P \geq 1$.

\section{APPENDIX C. Tables}


TABLE 1. Bias and mean square error (MSE) for all LPR-based estimators; $T=500$; using the parametric version of the bootstrap. (Unadjusted and analytically-bias-adjusted $L P R(P), P=0,1,2$; plus the bootstrap-bias-adjusted $L P R(P)-B B A(K)$; $K=1, \ldots, 3-P$, and their iteratively-adjusted ( $S S R)$ variants.) The lowest bias (in absolute value) and MSE for each design are highlighted in bold. Analogous figures for the ML estimator are reported in italics.

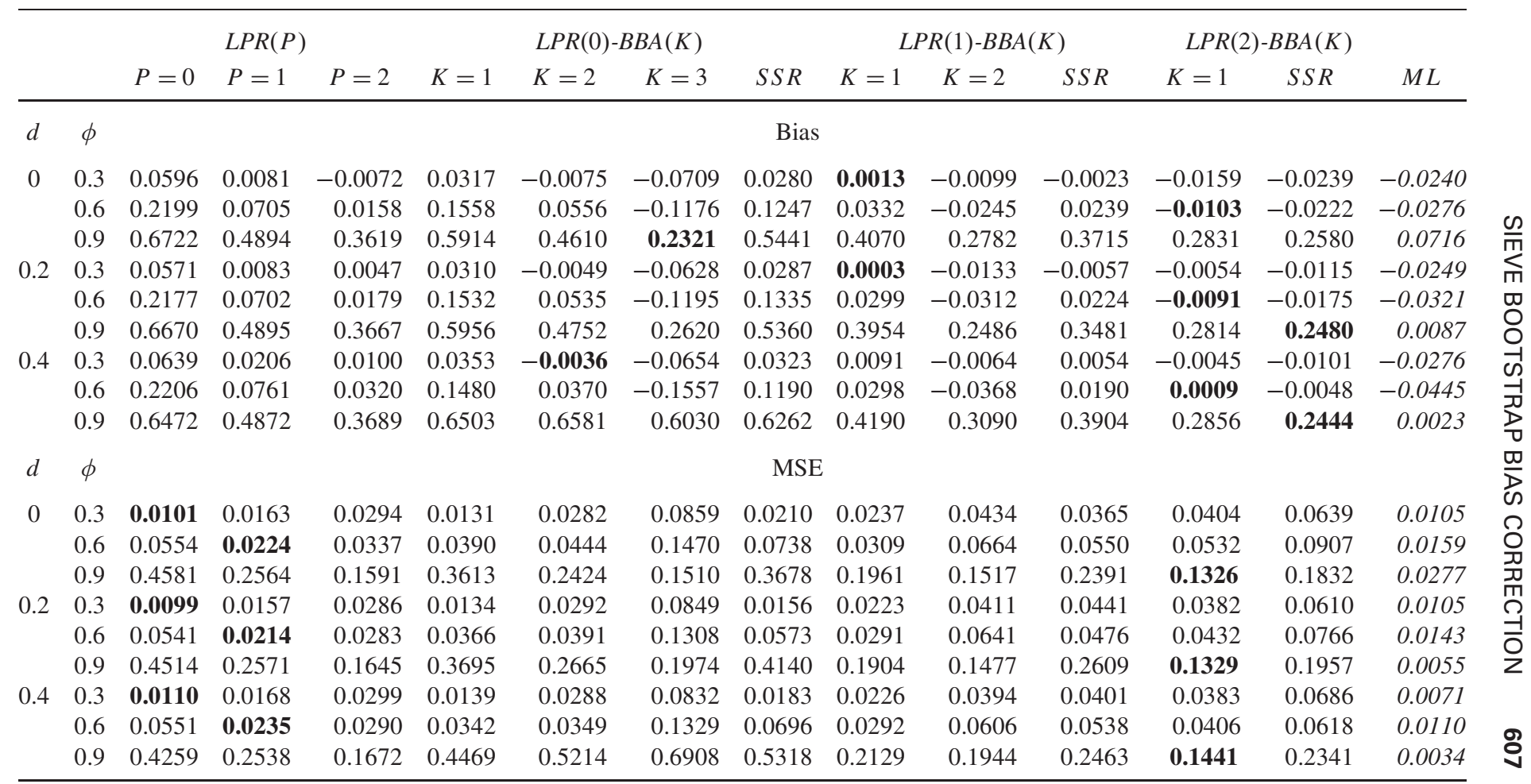


TABLE 2. Empirical coverage and length of nominal $95 \%$ bootstrap HPD intervals for unadjusted and analytically-bias-adjusted $L P R(P), P=0,1,2 ; T=500$; using the parametric version of the bootstrap. Analogous results for intervals based on the asymptotic distribution of each of the estimators are also displayed. Figures are averaged over all values of $d$ used in the experimental design for each value of $\phi$. The highlighting in bold indicates the empirical coverage closest to the nominal $95 \%$, and the shortest length.

\begin{tabular}{ccccccc}
\hline & \multicolumn{5}{c}{ BHDCI } & \multicolumn{3}{c}{ Asymp. interval } \\
& $P=0$ & $P=1$ & $P=2$ & $P=0$ & $P=1$ & $P=2$ \\
\cline { 2 - 7 }$\phi$ & & \multicolumn{7}{c}{ Coverage } \\
0.3 & 0.8980 & $\mathbf{0 . 9 5 7 3}$ & 0.9605 & 0.8348 & 0.9058 & 0.8885 \\
0.6 & 0.2405 & 0.9220 & $\mathbf{0 . 9 5 6 0}$ & 0.1738 & 0.8440 & 0.8735 \\
0.9 & 0.0000 & 0.0420 & $\mathbf{0 . 4 2 8 0}$ & 0.0000 & 0.0228 & 0.2693 \\
& & & & & & \\
$\phi$ & & & & & \\
0.3 & 0.3274 & 0.5267 & 0.6982 & $\mathbf{0 . 2 8 5 6}$ & 0.4283 & 0.5354 \\
0.6 & 0.3307 & 0.5272 & 0.6983 & $\mathbf{0 . 2 8 5 6}$ & 0.4283 & 0.5354 \\
0.9 & 0.3306 & 0.5222 & 0.6950 & $\mathbf{0 . 2 8 5 6}$ & 0.4283 & 0.5354 \\
\hline
\end{tabular}


TABLE 3. Bias and mean square error (MSE) for all LPR-based estimators*; $T=500$; using the nonparametric version of the bootstrap, with Gaussian and Student $t$ innovations. Figures are averaged over the four values of $d$ used in the experimental design for each value of $\phi$, and the lowest average bias (in absolute value) and MSE for each design highlighted in bold. Analogous figures for the (Gaussian) MLE are reported in italics. (* Unadjusted and analytically-bias-adjusted $L P R(P), P=0,1,2$, and the bootstrap-biasadjusted $L P R(P)-B B A(K) ; K=1, \ldots, 3-P$, plus their iteratively-adjusted (SSR) variants.)

\begin{tabular}{|c|c|c|c|c|c|c|c|c|c|c|c|c|c|}
\hline \multicolumn{14}{|c|}{ Gaussian innovations: Bias } \\
\hline 0.3 & 0.0609 & 0.0118 & 0.0086 & 0.0333 & -0.0048 & -0.0656 & 0.0322 & 0.0027 & -0.0108 & 0.0034 & -0.0004 & 0.0003 & -0.0257 \\
\hline 0.6 & 0.2209 & 0.0693 & 0.0269 & 0.1545 & 0.0525 & -0.1232 & 0.1365 & 0.0270 & -0.0366 & 0.0175 & -0.0027 & -0.0085 & -0.0349 \\
\hline 0.9 & 0.6650 & 0.4936 & 0.3709 & 0.6184 & 0.5409 & 0.3784 & 0.5833 & 0.4118 & 0.2837 & 0.3826 & 0.2866 & 0.2563 & 0.0219 \\
\hline 0.3 & 0.0103 & 0.0168 & 0.0300 & 0.0129 & 0.0268 & 0.0771 & 0.0145 & 0.0230 & 0.0389 & 0.0282 & 0.0377 & 0.0451 & 0.0096 \\
\hline 0.6 & 0.0554 & 0.0214 & 0.0307 & 0.0365 & 0.0373 & 0.1258 & 0.0528 & 0.0285 & 0.0610 & 0.0500 & 0.0449 & 0.0690 & 0.0134 \\
\hline 0.9 & 0.4490 & 0.2604 & 0.1677 & 0.4008 & 0.3560 & 0.3566 & 0.4387 & 0.2033 & 0.1652 & 0.2349 & 0.1384 & 0.2011 & 0.0103 \\
\hline \multicolumn{14}{|c|}{ Student $t$ innovations: Bias } \\
\hline 0.3 & 0.0626 & 0.0169 & 0.0098 & 0.0343 & -0.0050 & -0.0675 & 0.0308 & 0.0081 & -0.0055 & 0.0041 & 0.0004 & -0.0015 & -0.0264 \\
\hline \multicolumn{14}{|c|}{ Student $t$ innovations: MSE } \\
\hline 0.3 & 0.0105 & 0.0170 & 0.0292 & 0.0131 & 0.0274 & 0.0803 & 0.0183 & 0.0239 & 0.0425 & 0.0418 & 0.0368 & 0.0513 & 0.0111 \\
\hline 0.6 & 0.0563 & 0.0214 & 0.0297 & 0.0370 & 0.0376 & 0.1281 & 0.0634 & 0.0282 & 0.0604 & 0.0529 & 0.0438 & 0.0692 & 0.0142 \\
\hline 0.9 & 0.4503 & 0.2575 & 0.1669 & 0.4019 & 0.3586 & 0.3627 & 0.4472 & 0.2009 & 0.1647 & 0.2465 & 0.1380 & 0.2157 & 0.0101 \\
\hline
\end{tabular}

